\title{
Die Durchsetzung von Menschenrechten gegenüber Unternehmen mittels nichtfinanzieller Berichterstattung
}

Sebastian Eickenjäger*

\section{A. Einleitung}

Die 2011 vom UN Menschenrechtsrat verabschiedeten UN Guiding Principles on Business and Human Rights (UNGP) ${ }^{1}$ gelten heute als maßgeblicher Referenzpunkt für das Verhältnis zwischen Wirtschaft \& Menschenrechten. Die für Unternehmen enthaltenen Vorgaben der UNGP sind vor allem prozeduraler Natur und darauf ausgerichtet, Menschenrechte in die Strukturen von Unternehmen zu implementieren und diese zu einem »core business concern $\aleph^{2} \mathrm{zu}$ machen. Die Ausarbeitung eines Policy Commitments (Prinzipien 15 lit (a) und 16 UNGP) sowie die Durchführung von Due Diligence-Prozessen und Impact Assessments (Prinzipien 15, 17 und 18 UNGP) zielen darauf ab, die Ausgangssituation im Unternehmen $\mathrm{zu}$ evaluieren und davon ausgehend (schrittweise) die internen Strukturen auf Menschenrechte einzustellen. Das für den vorliegenden Beitrag zentrale Instrument ist die Praxis der nichtfinanziellen Berichterstattung, welche

* Dieser Beitrag wurde unterstützt durch den European Research Council (ERC, European Union Horizon 2020 research and innovation programme) im Rahmen des Projektes Transnational Force of Law [ERC-2014-CoG, No. 647313-Tansnational Force of Law, Andreas Fischer-Lescano]. Er basiert teilweise auf Eickenjäger, Menschenrechtsberichterstattung durch Unternehmen, 2017.

1 UN Special Representative of the Secretary-General on the Issue of Human Rights and Transnational Corporations and Other Business Enterprises (John Ruggie), Guiding Principles on Business and Human Rights: Implementing the United Nations "Protect, Respect and Remedy" Framework, Report of the Special Representative of the Secretary-General on the Issue of Human Rights and Transnational Corporations and other Business Enterprises, 21.3.2011, UN Doc. A/HRC/17/31. Kritisch hierzu Massoud, KJ 2013, 7; Blitt, TILJ 2012 (48), 33 (52 ff.) m.w.N; Bilchitz, IJHR 2012, 199; Simons, Journal of Human Rights and Environment 2012, 5; Hamm, KJ 2016, 479.

2 So Augenstein, Study of the Legal Framework on Human Rights and the Environment Applicable to European Enterprises Operating Outside the European Union, 2011, S. 75, Rn. 232 zur Zielrichtung nichtfinanzieller Berichterstattung. 
in den UNGP in den Prinzipien 16 lit. (d), 17 Satz 2 und 21 vorgesehen ist. Nichtfinanzielle Berichterstattung stellt einen Prozess zur Vermittlung von Informationen über soziale und ökologische Auswirkungen der Aktivitäten eines Unternehmens gegenüber interessierten Stakeholdern dar. Im Verhältnis zu den anderen in den UNGP genannten Instrumenten soll sie sicherstellen, dass diese umgesetzt bzw. implementiert werden. Durch die Richtlinie 2014/95/EU der Europäischen Union (EU) ${ }^{3}$ hat nichtfinanzielle Berichterstattung in Europa erheblich an Bedeutung gewonnen. Denn die Richtlinie sieht vor, dass die Mitgliedstaaten als groß geltende Unternehmen zur nichtfinanziellen Berichterstattung verpflichten müssen. Die Richtlinie 2014/95/EU ist dabei als ein Produkt der europäischen Strategie im Bereich der Corporate Social Responsibility (CSR) zu sehen. Die CSRStrategie sieht vor, dass Unternehmen zwar selbst bei der Fortentwicklung von CSR federführend sein sollen, nötigenfalls aber eine »intelligente Kombination « von Selbstregulierung und ergänzenden Legislativmaßnahmen vorzunehmen ist. ${ }^{4}$ In diesem Sinne wurde mit der Richtlinie eine legislative Maßnahme ergriffen, die darauf ausgerichtet ist, durch die Einführung einer obligatorischen Berichterstattung Impulse für unternehmerische Selbstregulierung zu setzen.

Im vorliegenden Kapitel soll der Frage nachgegangen werden, welchen Beitrag nichtfinanzielle Berichterstattung durch Unternehmen zur Durchsetzung von Menschenrechten gegenüber Unternehmen leisten kann. Die These des Beitrags lautet, dass Schwächen aktueller nichtfinanzieller Berichterstattung ohne Monitoringmechanismen nicht zu beheben sein werden und die Stärken des Instruments ohne entsprechende Mechanismen nicht entfaltet werden können. Ausgangspunkt des Beitrags ist die Umsetzung der Richtlinie 2014/95/EU in deutsches Recht. ${ }^{5}$ In einem ersten Schritt soll diese einer kritischen Würdigung unterzogen werden (B). Da-

3 Europäisches Parlament und Rat der Europäischen Union, Richtlinie 2014/95/EU des Europäischen Parlaments und des Rates vom 22.10.2014 zur Änderung der Richtlinie 2013/34/EU im Hinblick auf die Angabe nichtfinanzieller und die Diversität betreffender Informationen durch bestimmte große Unternehmen und Gruppen, 15.11.2014, ABl. L 330/1.

4 Europäische Kommission, Mitteilung der Kommission an das Europäische Parlament, den Rat, den Wirtschafts- und Sozialausschuss und den Ausschuss der Regionen - Eine neue EU-Strategie (2011-14) für die soziale Verantwortung der Unternehmen (CSR), 25.10.2011, KOM(2011) 681 endgültig, S. 9.

5 Das „Gesetz zur Stärkung der nichtfinanziellen Berichterstattung der Unternehmen in ihren Lage- und Konzernlageberichten (CSR-Richtlinie-Umsetzungsgesetz)“ 
ran anknüpfend soll die Praxis staatlicher Menschenrechtsberichterstattung betrachtet werden, um zu erfahren, welche Besonderheiten und Stärken bereits länger etablierte Praktiken der Menschenrechtsberichterstattung aufweisen (C). Es soll dabei herausgearbeitet werden, dass die fortlaufende Ein- und Anleitung von Lernprozessen eine wesentliche Stärke von auf Berichterstattung setzenden Monitoringverfahren ist (D). Der Gesetzesentwurf sieht die Einrichtung von Verfahren, die auf ein Monitoring bzgl. der Umsetzung der Maßnahmen gerichtet sind, über die berichtet wird, nicht vor. ${ }^{6}$ Deshalb sollen zuletzt Perspektiven aufgezeigt werden, wie und wo entsprechende Verfahren eingerichtet werden könnten (E).

\section{B. Umsetzung der Richtlinie 2014/95/EU in deutsches Recht}

Bisher handelte es sich bei der nichtfinanziellen Berichterstattung in Deutschland um eine auf Freiwilligkeit beruhende Praxis. Mit der Umsetzung der Richtlinie 2014/95/EU in deutsches Recht ${ }^{7}$ wurden insb. eine Änderung der Regelungen des HGB zum Lagebericht und Konzernlagebericht vorgenommen, mit der nichtfinanzielle Berichterstattung nunmehr in Deutschland für große Unternehmen verbindlich ist. Ziel der Richtlinie

wurde in der Fassung des Ausschusses für Recht und Verbraucherschutz (BT-Drs. 18/11450 v. 8.3.2017) vom Bundestag angenommen und Anfang April 2017 verkündet (BGB1. I 2017 S. 802).

6 Siehe hierzu unten B. VIII.

7 Siehe hierzu bzw. zur Richtlinie auch Bortenlänger / von Altenbockum, ZGG 2014, 87; Böcking, DB 2017, M5; Kajüter IRZ 2016, 507; Kajüter, DB 2017, 617; Kumm / Woodtli, DK 2016, 218; Meeh-Bunse / Hermeling / Schomaker, DStR 2016, 2769; Müller, BB 2016, 1; Müller / Stawinoga / Velte, ZfU 2015, 313; Nietsch, NZG 2016, 1330; Nietsch / Munerotto, CB 2016, 177; Roth-Mingram, NZG 2015, 1341; Seibt, DB 2016, 2707; Spießhofer, NZG 2014, 1281; Stawinoga / Velte, DB 2016, 841; Voland, DB 2014, 2815. Siehe auch die zahlreichen Stellungnahmen zum Gesetzgebungsverfahren (abrufbar unter www.bmjv.de/SharedDocs/Gesetzgeb ungsverfahren/DE/CSR-Richtlinie-Umsetzungsgesetz.html) und die Stellungnahmen vor dem Ausschuss des Bundestags für Recht und Verbraucherschutz. Siehe auch die Beiträge von Horst, Segger und Brunk in diesem Band. Zur Diskussion, ob mit der Verabschiedung der Richtlinie 2014/95/EU eine Abkehr vom ShareholderValue-Konzept hin zu einem Stakeholder-Value-Ansatz zu sehen ist, siehe Hommelhoff, Festschrift für Gerrick Freiherr v. Hoyningen-Huene zum 70. Geburtstag, 2014, S. 137; Hommelhoff, Festschrift für Bruno M. Kühler zum 70. Geburtstag, 2015, S. 291; Hommelhoff, NZG 2015, 1329; Schön, ZHG 2016, 279; Seibt, DB 2016, 2707. 
und der Umsetzung ist es, »Regelungen zu treffen, die das Vertrauen von Investoren sowie Verbraucherinnen und Verbrauchern in Unternehmen stärken sollen, indem mehr als bisher über nichtfinanzielle Aspekte der Tätigkeit von bestimmten Unternehmen berichtet wird. $\aleph^{8}$ Mittelbar soll dadurch auch das Verhalten von Unternehmen im Hinblick auf Menschenrechte und andere von der Berichterstattungspflicht umfasste nichtfinanzielle Belange beeinflusst werden, indem mit der Pflicht zur Berichterstattung Impulse geliefert werden, entsprechenden Belange im Geschäftsalltag zu berücksichtigen. ${ }^{9}$

Das Umsetzungsgesetz zielt auf eine weitgehende 1:1-Umsetzung der Richtlinie ab. In der Folge sollen die wesentlichen Aspekte des Gesetzes sowie die diesbezüglich (insbesondere im Gesetzgebungsverfahren) vorgebrachte Kritik hieran dargestellt werden.

\section{Persönlicher Anwendungsbereich}

Ein erster Streitpunkt war zunächst die Frage nach dem persönlichen Anwendungsbereich. ${ }^{10}$ Gemäß $§ 289$ b Abs. 1 S. 1 HGB müssen Kapitalgesellschaften (einschließlich haftungsbeschränkter Personengesellschaften) bzw. nach $\S 315$ b Abs. 1 S. 1 HBG Kapitalgesellschaften, die Mutterunternehmen sind, ihren Lagebericht bzw. Konzernlagebericht um eine nichtfinanzielle Erklärung erweitern, wenn sie kapitalmarktorientiert im Sinne des $\S 264 \mathrm{~d}$ HBG sind, im Jahresdurchschnitt mehr als 500 Arbeitnehmer_innen beschäftigen und entweder eine Bilanzsumme von 20.000.000 Euro oder Umsatzerlöse in den zwölf Monaten vor dem Abschlussstichtag

8 Bundesregierung der Bundesrepublik Deutschland, Entwurf eines Gesetzes zur Stärkung der nichtfinanziellen Berichterstattung der Unternehmen in ihren Lageund Konzernlageberichten (CSR-Richtlinie-Umsetzungsgesetz), 17.10.2016, Drs. 18/9982, S. 24.

9 Ebenda.

10 Die Frage des Anwendungsbereichs war bereits ein zentraler Streitpunkt im Rahmen der Verhandlungen um die Richtlinie. Siehe hierzu Kinderman, WSI-Mitteilungen 2015, 2015, S. 613. Der weit gefasste Richtlinienentwurf wurde dementsprechend abgeändert, womit die Zahl der von der Berichterstattungspflicht betroffenen Unternehmen schätzungsweise von ca. 18.000 auf ca. nunmehr 6.000 gesunken ist (ebenda, S. 616; Glaser, IRZ 2015, 55 (56)). 
von 40.000.000 Euro aufweisen. ${ }^{11}$ Kleine und mittlere Unternehmen sind danach zunächst von der Berichtspflicht befreit. Dem Erwägungsgrund (ErwG) 14 der Richtlinie 2014/95/EU ist zwar zu entnehmen, dass die Mitgliedstaaten sich nicht daran gehindert sehen sollen, den Geltungsbereich ihrer Gesetzgebung weiter zu fassen. Es wird dort jedoch gleichsam betont, dass kleine und mittlere Unternehmen grds. von der Pflicht befreit werden sollten, um diese vor unverhältnismäßigen administrativen und finanziellen Belastungen zu bewahren. ${ }^{12}$

Im Gesetzgebungsverfahren wurde diesbezüglich vor allem kritisiert, dass nur kapitalmarktorientierte Kapitalgesellschaften, haftungsbeschränkte Personengesellschaften, Kreditinstitute und Versicherungsunternehmen von der Berichtspflicht umfasst sind. Gleichzeitig seien aber große, global agierende nicht-kapitalmarktorientierte Unternehmen, die keiner dieser Kategorien zugeordnet werden können, von der Pflicht ausgenommen, obwohl im Hinblick auf deren Größe und deren Aktivitäten keine wesentlichen Unterschiede zu den umfassten Unternehmen bestehen. ${ }^{13}$

11 Über die Verweise in $\S 340$ Abs. 1 a und $\S 340$ a Abs. 1 a HGB sind auch Kreditinstitute und Versicherungsunternehmen, die die oben genannten Kriterien erfüllen, berichtspflichtig.

12 Spießhofer weist zutreffend darauf hin, dass mittelbar auch Unternehmen, die sich örtlich außerhalb des Anwendungsbereiches der Richtlinie befinden, sowie kleine und mittlere Unternehmen von der Berichterstattungspflicht betroffen sein können (Spießhofer, NZG 2014, 1281 (1283)). Dies ergibt sich aus § 289 c HGB, wonach auch über die wesentlichen Risiken, die mit der Geschäftstätigkeit des Unternehmens - einschließlich, wenn dies relevant und verhältnismäßig ist, seiner Geschäftsbeziehungen, seiner Erzeugnisse oder seiner Dienstleistungen - verknüpft sind, berichtet werden soll. Denn die geforderten Informationen im Zusammenhang mit den Geschäftsbeziehungen eines Unternehmens werden in der Regel durch Audits oder Offenlegungsanforderungen zwischen den Unternehmen generiert (ebenda).

13 Germanwatch, Stellungnahme zum Regierungsentwurf eines Gesetzes zur Stärkung der nichtfinanziellen Berichterstattung der Unternehmen in ihren Lage- und Konzernlageberichten, 2016, S. 4; Felber, Stellungnahme zum Entwurf des Gesetzes zur Stärkung der nichtfinanziellen Berichterstattung der Unternehmen in ihren Lage- und Konzernlageberichten, 2016, S. 1; Fraktion BÜNDNIS 90/DIE GRÜNEN et al., Antrag Zukunftsfähige Unternehmensverantwortung - Nachhaltigkeitsberichte wirksam und aussagekräftig ausgestalten - Umsetzung der CSRRichtlinie, 19.10.2016, BT Drs. 18/10030, S. 4. 


\section{Inhaltliche Anforderungen}

In inhaltlicher Hinsicht werden Unternehmen gemäß § 289 c Abs. 2 HBG verpflichtet, über Umweltbelange, Arbeitnehmer_innenbelange, Sozialbelange, die Achtung der Menschenrechte und die Bekämpfung von Bestechung und Korruption zu berichten. § 289 Abs. 3 HGB gibt nähere Anweisungen für die Berichterstattung zu diesen Aspekten. Dort heißt es, dass diesbezüglich in der nichtfinanziellen Erklärung diejenigen Angaben zu machen sind, die für das Verständnis des Geschäftsverlaufs, des Geschäftsergebnisses, der Lage der Kapitalgesellschaft sowie der Auswirkungen ihrer Tätigkeit auf die in Absatz 2 genannten Aspekte erforderlich sind. Im Einzelnen werden diese Anforderungen in Abs. 3 Nr. 1 bis 6 nochmals wie folgt konkretisiert: Erforderlich ist die Beschreibung

1. der von der Kapitalgesellschaft verfolgten Konzepte, einschließlich der von der Kapitalgesellschaft angewandten Due-Diligence-Prozesse,

2. der Ergebnisse der Konzepte nach Nummer 1,

3. der wesentlichen Risiken, die mit der eigenen Geschäftstätigkeit der Kapitalgesellschaft verknüpft sind und die sehr wahrscheinlich schwerwiegende negative Auswirkungen auf die in Absatz 2 genannten Aspekte haben oder haben werden, sowie die Handhabung dieser Risiken durch die Kapitalgesellschaft,

4. der wesentlichen Risiken, die mit den Geschäftsbeziehungen der Kapitalgesellschaft, ihren Produkten und Dienstleistungen verknüpft sind und die sehr wahrscheinlich schwerwiegende negative Auswirkungen auf die in Absatz 2 genannten Aspekte haben oder haben werden, soweit die Angaben von Bedeutung sind und die Berichterstattung über diese Risiken verhältnismäßig ist, sowie die Handhabung dieser Risiken durch die Kapitalgesellschaft,

5. der bedeutsamsten nichtfinanziellen Leistungsindikatoren, die für die Geschäftstätigkeit der Kapitalgesellschaft von Bedeutung sind,

6. soweit es für das Verständnis erforderlich ist, Hinweisen auf im Jahresabschluss ausgewiesene Beträge und zusätzliche Erläuterungen dazu.

Im Gesetzgebungsverfahren wurde diesbezüglich kritisiert, dass keine 1:1Umsetzung vorgenommen wurde. Der Bundesrat empfahl etwa in seiner Stellungnahme, eine vorgenommene Verschärfung des Wortlauts zurückzunehmen. Denn schon im Gesetzesentwurf hieß es in $\S 289$ c Abs. 3 Nr. 3 und $4 \mathrm{HGB}$, dass über "sehr wahrscheinlich schwerwiegende negative Auswirkungen" berichtet werden soll, während in der Richtlinie nur von 
"wahrscheinlich negativen Auswirkungen" die Rede ist. ${ }^{14}$ Durch diese Einschränkung wird der Umfang der Berichterstattung unangemessen eingeschränkt und die ohnehin schon durch unbestimmte Rechtsbegriffe bestimmte Regulierung durch eine weitere Aufweichung relativiert. Es wird zudem befürchtet, dass der Wortlaut des Abs. 3 so ausgelegt werden kann, dass »schwere und andauernde Menschenrechtsverletzungen oder die andauernde Verseuchung von Naturschutzgebieten und der dort lebenden Menschen (...) nur offengelegt werden [müssen], wenn sie sich auch auf das Geschäftsergebnis auswirken oder für das Verständnis des Geschäftsverlaufs erforderlich sind «. ${ }^{15}$ Eine solche Auslegung würde die Berichtspflicht zusätzlich erheblich einengen. ${ }^{16}$

\section{Berichterstattungsstandards}

Die inhaltlichen Vorgaben zur Berichtspflicht geben zwar vor, welche Informationen offenzulegen sind. Eine detaillierte Anleitung für die Erfassung und Präsentation der Informationen ist den Vorgaben indes nicht zu entnehmen. ${ }^{17} \S 289 \mathrm{~d} \mathrm{HBG}$ weist diesbezüglich darauf hin, dass Unternehmen für die Erstellung der nichtfinanziellen Erklärung nationale, europäische oder internationale Rahmenwerke nutzen können und angeben müs-

14 Bundesrat, Stellungnahme des Bundesrates zum Entwurf des CSR-Richtlinie-Umsetzungsgesetzes, 04.11.2016, Drs. 547/16.

15 Fraktion BÜNDNIS 90/DIE GRÜNEN et al., Antrag Zukunftsfähige Unternehmensverantwortung - Nachhaltigkeitsberichte wirksam und aussagekräftig ausgestalten - Umsetzung der CSR-Richtlinie, 19.10.2016, BT Drs. 18/10030, S. 5.

16 Eingefordert wurde deshalb eine Korrektur des Wortlauts bzw. eine Klarstellung, dass eine entsprechende Konnexität nicht erforderlich ist, etwa durch eine Formulierung des Absatz 3, die wie folgt gefasst ist: „Zu den in Absatz 2 genannten Aspekten sind in der nichtfinanziellen Erklärung sowohl jeweils die Angaben zu machen, die für das Verständnis des Geschäftsverlaufs, des Geschäftsergebnisses, der Lage der Kapitalgesellschaft als auch diejenigen, die für das Verständnis der Auswirkungen ihrer Tätigkeit auf die in Absatz 2 genannten Aspekte erforderlich sind, einschließlich [...].“ Germanwatch, Stellungnahme zum Regierungsentwurf eines Gesetzes zur Stärkung der nichtfinanziellen Berichterstattung der Unternehmen in ihren Lage- und Konzernlageberichten, 2016, S. 3.

17 Spießhofer weist zudem darauf hin, dass Unklarheit bzgl. der Frage besteht, ob in dem Fall, dass ein Unternehmen einen von dem Bericht über finanzielle Informationen separate nichtfinanzielle Erklärung abgibt, eine Pflicht zur Heranziehung entsprechender Standards besteht (Spießhofer, NZG 2014, 1281 (1286)). 
sen, welches Rahmenwerk sie benutzt haben. ${ }^{18}$ Mit der Regelung wurde es damit verpasst, Unternehmen einheitliche Berichtsstandards verbindlich vorzugeben. Während vor allem Unternehmensverbände die durch diese Regelung überlassenen Spielräume ausdrücklich begrüßen, wird in diesem Zusammenhang zutreffend auf die erhebliche Heterogenität der Rahmenwerke im Hinblick auf deren Ausgestaltung und Anforderungen hingewiesen. Es wird bemängelt, dass mit dieser Regelung eine Harmonisierung der Berichtspraxis in der EU »auf tönernen Füßen« stehe und eine Vergleichbarkeit der Berichte nicht gewährleistet erscheine. ${ }^{19}$ Die dadurch nach wie vor bestehenden Unterschiede in der Berichterstattung führen zu Unsicherheiten bei den Leser_innen der Berichte. Art. 2 RL 2014/95/EU ist zwar der an die Kommission gerichtete Auftrag zu entnehmen, »Leitlinien zur Methode der Berichterstattung über nichtfinanzielle Informationen « zu erstellen. Allerdings wird es sich bei diesen Leitlinien gemäß Art. 2 RL 2014/95/EU ausdrücklich nur um unverbindliche Vorgaben handeln, so dass auch hierdurch keine Vereinheitlichung der Berichterstattung $\mathrm{zu}$ erwarten ist.

\section{Ausnahmen von der Berichtspflicht}

Die Berichtspflicht gilt zudem nicht absolut. Soweit ein Unternehmen in Bezug auf einen oder mehrere der benannten Belange kein Konzept verfolgt, hat die nichtfinanzielle Erklärung gemäß § 289 c Abs. 4 HBG dies anstelle der auf den jeweiligen Aspekt bezogenen Angaben in der nichtfinanziellen Erklärung klar und begründet zu erläutern..$^{20}$ Die damit statuierte Report or Explain-Regelung wird allgemein als sinnvoller Mechanismus bewertet, der einer heterogenen Praxis und Ausrichtung der betroffe-

18 Die bekanntesten von Internationalen Organisationen angestoßenen Initiativen betreffend nichtfinanzielle Berichterstattung sind die OECD Guidelines for Multinational Enterprises, die ISO 26000 und die United Nations Global Compact Communication on Progress (UNGC COP). Von besonderer Bedeutung sind daneben Initiativen privater Akteur_innen, wie die Leitfäden der Global Reporting Initiative (GRI), des Carbon Disclosure Projects (CDP) und der Human Rights Reporting and Assurance Frameworks Initiative (RAFI).

19 Spießhofer, NZG 2014, 1281 (1285). So auch Roth-Mingram, NZG 2015, 1341 (1343).

20 Siehe zudem die in $\S 289$ b Abs. 2 und $\S 315$ b Abs. 2 HBG vorgesehen Ausnahmen. 
nen Unternehmen entgegenkommt und einen flexiblen und einzelfallbezogenen Umgang mit der Berichtspflicht ermöglicht.

Eine weitere Ausnahme für die Berichtspflicht sieht $\S 289$ e Abs. 1 Nr. 1 HGB für bestimmte Informationen vor. Danach müssen in die nichtfinanzielle Erklärung ausnahmsweise keine Angaben zu künftigen Entwicklungen oder Belangen, über die Verhandlungen geführt werden, aufgenommen werden, wenn die Angaben nach vernünftiger kaufmännischer Beurteilung der Mitglieder des vertretungsberechtigten Organs des Unternehmens geeignet sind, dem Unternehmen einen erheblichen Nachteil zuzufügen. Einschränkend gilt diesbezüglich gemäß $§ 289$ e Abs. 1 Nr. 2 HGB, dass das Weglassen der Angaben ein den tatsächlichen Verhältnissen entsprechendes und ausgewogenes Verständnis des Geschäftsverlaufs, des Geschäftsergebnisses, der Lage des Unternehmens und der Auswirkungen seiner Tätigkeit nicht verhindern darf. ${ }^{21}$ Der Zweck dieser Ausnahmeregelung besteht darin, die wirtschaftlichen Interessen der Unternehmen $\mathrm{zu}$ schützen, indem bestimmte Informationen von der Berichtspflicht ausgenommen sind. An dieser Regelung wird indes zu Recht kritisiert, dass sie zu unbestimmt ist, den Entscheider_innen zu viel Spielraum überlässt und zudem wie ein Wesentlichtlichkeitsfilter wirken kann, wonach Informationen zu den geforderten Aspekten nicht offengelegt werden müssen, wenn die Informationen nach Ansicht des Unternehmens keinen Einfluss auf die Situation des Unternehmens haben. ${ }^{22}$

Damit sich ein Unternehmen auf die zuvor benannte Ausnahme berufen kann, muss eine »vernünftige kaufmännische Beurteilung der Mitglieder des vertretungsberechtigten Organs des Unternehmens « vorliegen. ${ }^{23}$ Diese Formulierung ist dahingehend auszulegen, dass in entsprechenden Fällen tatsächlich eine begründete Erklärung über die Nichtvorlage bestimmter Informationen vorgelegt werden muss. Wird eine Information aus berechtigten Gründen zurückgehalten, muss in der nichtfinanziellen Erklärung oder dem gesonderten Bericht ein Hinweis darauf enthalten sein, der die

21 Gemäß $\S 289$ e Abs. 2 HGB sind die Angaben in die darauffolgende nichtfinanzielle Erklärung aufzunehmen, wenn die Gründe für die Nichtaufnahme der Angaben nach der Veröffentlichung der nichtfinanziellen Erklärung entfallen.

22 Germanwatch, Stellungnahme zum Regierungsentwurf eines Gesetzes zur Stärkung der nichtfinanziellen Berichterstattung der Unternehmen in ihren Lage- und Konzernlageberichten, 2016, S. 7 f.

23 In Art. 19 a Abs. 1 UAbs. 4 und Art. 29 a Abs. 1 UAbs. 4 BilanzRL heisst es, dass eine „ordnungsgemäß begründete Einschätzung der Mitglieder der Verwaltungs-, Leitungs- und Aufsichtsorgane" vorliegen muss. 
Gründe der Nichtveröffentlichung angibt und den Gehalt der Informationen so konkret wie möglich skizziert. Denn wenn bestimmte Informationen zurückgehalten würden, ohne dies nach außen zu kommunizieren, wäre insgesamt niemals nachvollziehbar, ob die offengelegten Informationen tatsächlich vollständig sind.

\section{Externe Prüfung der Berichterstattung}

Einen weiteren Streitpunkt bildet die Regelung des Gesetzesentwurfs zur inhaltlichen externen Prüfung der Berichterstattung. Im Bereich der finanziellen Berichterstattung sind Wirtschaftsprüfer_innen und Wirtschaftsprüfungsgesellschaften aufgrund gesetzlicher Verpflichtungen zur externen Prüfung der Rechnungslegung bereits fester Bestandteil. Die auf globaler Ebene im Bereich der finanziellen Berichterstattung etablierten Wirtschaftsprüfungsgesellschaften haben mittlerweile auch die nichtfinanzielle Berichterstattung als neuen Markt für sich entdeckt. ${ }^{24}$ Hauptantrieb für die verstärkte Nachfrage nach externer Prüfung für Berichte über nichtfinanzielle Informationen ist das zunehmende Interesse von Investor_innen und Konsument_innen an verlässlichen und belastbaren nichtfinanziellen Informationen. ${ }^{-25}$

Die Änderungen des HGB sehen eine Prüfungspflicht grds. nicht vor. Wenn aber eine externe Prüfung freiwillig durchgeführt wurde, sind die Ergebnisse gemäß $§ 289$ b Abs. 4 HGB zu veröffentlichen. ${ }^{26}$ Auf der einen Seite wird kritisiert, dass Informationen nur verlässlich und glaubwürdig sind, wenn sie unabhängig geprüft wurden; im Anschluss daran wird daher eine Pflicht zur Durchführung externer Prüfungen gefordert. ${ }^{27}$ Auf der anderen Seite wird die Ansicht vertreten, dass die im Gesetzesentwurf vorge-

24 Die Global Reporting Initiative weist darauf hin, dass im Jahre 2012 über 46\% aller Berichte, die in ihrer Datenbank gelistet sind, in irgendeiner Weise durch Dritte geprüft wurden und sich global immer mehr ein Trend in Richtung externer Prüfung verzeichnen lässt (Global Reporting Initiative (GRI) The External Assurance of Sustainability Reporting, 2013, S. 7).

25 Wallage, Auditing 2000 (19.1), 53 (53 f).

26 Diese Regelung tritt allerdings erst am 1.1.2019 in Kraft (Art. 12, BT-Drs. $18 / 11450$ v. 8.3.2017).

27 Germanwatch, Stellungnahme zum Regierungsentwurf eines Gesetzes zur Stärkung der nichtfinanziellen Berichterstattung der Unternehmen in ihren Lage- und Konzernlageberichten, 2016, S. 6 f. 
sehene Veröffentlichungspflicht letztlich Unternehmen eher davon abhalten wird, ihre Berichte prüfen zu lassen. ${ }^{28}$ Es wird zudem darauf hingewiesen, dass die internen Berichtsprozesse und Systeme in der Regel noch nicht in ausreichendem Maße auf die Berichtsprozesse im Bereich der nichtfinanziellen Berichterstattung eingestellt sind. ${ }^{29}$

Auch wenn die externe Prüfung einen wertvollen Beitrag zur Verifizierung und Überprüfung der Berichterstattung leisten kann, begegnet die Praxis bisher nicht unerheblichen Herausforderungen. Zum einen bewirkt die Vielzahl an verfügbaren und verwendeten Berichts- und Überprüfungsstandards nicht nur Unsicherheiten in Bezug auf die Ergebnisse des Prüfungsprozesses, sondern führt oftmals auch dazu, dass Leser_innen gegenüber den Prüfungsberichten mit der Praxis bzw. der Ausrichtung der externen Prüfung nicht konvergierende Erwartungen entwickeln. ${ }^{30}$ Zudem kommt die Prüfungspraxis schnell an ihre Grenzen, wenn es darum gehen soll zu prüfen, ob und inwieweit das Unternehmen z.B. Due DiligenceProzesse und Impact Assessments durchführt, zu welchen Ergebnissen solche Prozesse in aktuellen und evtl. kritischen Fällen gekommen sind und inwiefern hier Verbesserungsbedarf besteht. Zu Recht wird in diesem Zusammenhang kritisiert, dass Prüfungsberichte in der Regel zwar angeben, dass Menschenrechtsmechanismen bestehen und auch weiterentwickelt wurden, jedoch selten Aufschluss darüber geben, ob diese auch tat-

28 Institut der Wirtschaftsprüfer in Deutschland (IDW), Stellungnahme zu dem Regierungsentwurf eines Gesetzes zur Stärkung der nichtfinanziellen Berichterstattung der Unternehmen in ihren Lage- und Konzernlageberichte, 2016, S. 2. In diesem Zusammenhang weist die Human Rights Reporting and Assurance Frameworks Initiative (RAFI) darauf hin, dass es für solche Unternehmen, die sich in einer Startphase der Berichterstattung und Implementierung von menschenrechtsorientierten Mechanismen befinden, sinnvoll sein kann, den Fokus auf interne Prüfungen zu legen oder externe Prüfer_innen zu beauftragen, ohne eine öffentliche Kommunikation zu vereinbaren (Human Rights Reporting and Assurance Frameworks Initiative (RAFI), Visions for Human Rights Assurance, 2015, S. 4).

29 Institut der Wirtschaftsprüfer in Deutschland e.V., Konzept zur Umsetzung der CSR-Richtlinie, 2015, S. 1 f., 8 f.; Deutscher Genossenschafts- und Raiffeisenverband e.V., Stellungnahme zum BMJV-Konzept zur Umsetzung der CSR-Richtlinie, 2015, S. 3.

30 Schelluch / Gay beschreiben dieses Phänomen als Expectation Gap, dem Auseinanderfallen von Erwartungen an das Auditing und dem tatsächlichen Gegenstand der Prüfung (Schelluch / Gay, Accounting \& Finance 2006, 653). 
sächlich effektiv sind. ${ }^{31}$ Um eine wirksame Überprüfung der Menschenrechtsbilanz eines Unternehmens vornehmen zu können, müsste in der Prüfung ein Wandel von einer technischen Überprüfung von Fakten und Informationen hin zu einer umfassenden Prüfung und einer an menschenrechtlichen Instrumenten orientierten Evaluation der Strukturen und Prozesse des Unternehmens stattfinden. ${ }^{32}$ Eine weitere Schwachstelle externer Prüfung besteht darin, dass allein die Unternehmen bestimmen, was Gegenstand der Prüfung sein soll und dass Prüfer_innen in einem wirtschaftlichen Abhängigkeitsverhältnis zu den zu prüfenden Unternehmen stehen.

\section{Form und Zeitpunkt der Berichterstattung}

Im Hinblick auf die Form und den Zeitpunkt der Berichterstattung sieht der Entwurf vor, dass die nichtfinanzielle Erklärung entweder als Teil des (konsolidierten) Lageberichts ( $\$ 289$ b Abs. 1 HGB) oder als separate Erklärung zusammen mit dem Lagebericht oder spätestens sechs Monate nach dem Abschlussstichtag auf der Homepage des Unternehmens ( 2289 b Abs. 3 HGB) veröffentlicht werden soll. Kritisiert wird, dass diese Regelung konträr zum Ansatz des sog. Integrated Reporting stehe, dessen Ausgangspunkt die Annahme ist, dass sich erst aus der Gesamtschau der finanziellen, ökologischen und sozialen Bilanz eines Unternehmens eine Einschätzung über den Geschäftsverlauf, das Geschäftsergebnis und die Lage eines Unternehmens vornehmen lässt. ${ }^{33}$

\section{Prüfungspflicht des Aufsichtsrats und Sanktionen}

Gemäß 170 Abs. 1 S. 3 AktG sind nunmehr auch der (Konzern)Lagebericht inklusive der nichtfinanziellen Erklärung bzw. der gesondert erstellte

31 Human Rights Reporting and Assurance Frameworks Initiative (RAFI), Visions for Human Rights Assurance, 2015, S. 4.

32 Kocher und Wenckebach plädieren in diesem Zusammenhang dafür, die Entwicklung eines eigenständigen Marktes unabhängiger Prüfung nichtfinanzieller Berichterstattung zu fördern (Kocher / Wenckebach, KJ 2013, 18 (27)).

33 Siehe etwa Fraktion BÜNDNIS 90/DIE GRÜNEN et al., Antrag Zukunftsfähige Unternehmensverantwortung - Nachhaltigkeitsberichte wirksam und aussagekräftig ausgestalten - Umsetzung der CSR-Richtlinie, 19.10.2016, BT Drs. 18/10030, S. 5. 
nichtfinanzielle Bericht vom Vorstand dem Aufsichtsrat vorzulegen. Mit dem neu eingefügten $\S 171$ Abs. 1 S. 4 AktG erstreckt sich die Prüfungspflicht des Aufsichtsrats auch auf die nichtfinanzielle Erklärung als Teil des (Konzern)Lageberichts sowie den gesondert erstellten nichtfinanziellen Bericht. ${ }^{34}$

Das Umsetzungsgesetz sieht zudem die Ausweitung des HGB-Sanktionssystems auf den Bereich der nichtfinanziellen Berichterstattung und eine erhebliche Erweiterung des Sanktionsrahmens bzgl. der einschlägigen Tatbestände vor. ${ }^{35}$

\section{Monitoringmechanismen}

Neben den genannten Regelungen zur externen Prüfung und der Prüfungspflicht des Aufsichtsrats sieht der Gesetzesentwurf keine Verfahren zum Monitoring oder zur Evaluation der Berichterstattung vor. Die institutionelle Überprüfung der Berichterstattung wird in der Richtlinie lediglich in ErwG 10 thematisiert. Dort heißt es, dass die Mitgliedstaaten sicherstellen sollen, »dass es sachgerechte und wirksame Mechanismen gibt, mit denen die Angabe nichtfinanzieller Informationen durch Unternehmen im Einklang mit dieser Richtlinie gewährleistet wird. Zu diesem Zweck sollten die Mitgliedstaaten dafür sorgen, dass wirksame nationale Verfahren eingerichtet sind, mit denen die Erfüllung der Pflichten nach dieser Richtlinie gewährleistet wird«. ErwG 10 der Richtlinie enthält damit keine Angaben, wie die »nationalen Verfahren « konkret ausgestaltet sein sollten, insbesondere nicht zu der Frage, aus welchem Anlass ein entsprechendes Verfahren eingeleitet werden und bei welchen Institutionen es angesiedelt sein sollte. Es wird allein weiter konkretisiert, dass diese Verfahren »allen natürlichen und juristischen Personen offenstehen (sollten), die gemäß nationalem Recht ein berechtigtes Interesse daran haben, dass sichergestellt wird, dass die Bestimmungen dieser Richtlinie eingehalten werden «.

Für Verfahren zum Monitoring der Berichterstattung spricht zunächst, dass ansonsten die Prüfung der Berichte allein im Ermessen der Unterneh-

34 Ausführlich hierzu Hennrichs / Pöschke, NZG 2017, 121; Kumm / Woodtli, DK 2016, 218 (228f.). Siehe auch folgende kritische Stellungnahme: Arbeitskreis Bilanzrecht Hochschullehrer Rechtswissenschaft, NZG 2016, 1337.

35 Hierzu ausführlich Seibt, DB 2016, 2707 (2714); Kumm / Woodtli, DK 2016, 218 (229 ff.). 
men steht oder davon abhängig ist, dass zivilgesellschaftliche Akteur_innen die Berichterstattung eines Unternehmens in den Blick nehmen und ggf. thematisieren. Bei der Behandlung der Berichterstattung eines Unternehmens durch zivilgesellschaftliche Akteur_innen besteht das Problem, dass kein Verfahren und keine unabhängige Instanz für die Beurteilung der streitigen Fragen und für eine inhaltliche Auseinandersetzung bereitstehen. Es fehlt die Möglichkeit, Vorwürfe in einem hierfür vorgesehenen Verfahren auszuräumen. Und es bestehen vor allem dann, wenn ein Unternehmen sich der Auseinandersetzung mit Vorwürfen verweigert, bisher nur unzureichende prozedurale Möglichkeiten, Missstände in der Berichterstattung bzw. der Umsetzung des Gegenstands der Berichterstattung aufzuarbeiten oder auf eine Richtigstellung der Berichterstattung hinzuwirken. Die Wirkung nichtfinanzieller Berichterstattung ist zudem kaum messbar. Es ist nicht nachvollziehbar, ob die getätigten Angaben richtig und umfassend sind und ob Unternehmen im Rahmen der Berichterstattung Menschenrechte in ihren Strukturen implementieren, d.h. Konzepte wie Due Diligence-Prozesse, Stakeholder Engagement, Impact Assessments und interne Beschwerdemechanismen tatsächlich umsetzen.

Eine andere Folge des Mangels an Monitoringmechanismen ist das Phänomen des Greenwashing. Der Begriff des Greenwashing beschreibt das Problem, dass Unternehmen Freiräume in der Berichterstattung oder der Darstellung ihrer Aktivitäten häufig nutzen, um ihr Image durch die Hervorhebung umwelt- oder sozialfreundlicher Programme oder Produkte des Unternehmens, bzw. durch die Unterschlagung von Informationen zu eigenen sozial- oder umweltschädlichen Praktiken, aufzubessern. Entsprechende Kampagnen und Strategien sind darauf ausgerichtet, tatsächliche Problemlagen dadurch zu verschleiern, dass eine idealisierte Version des eigenen Unternehmens öffentlichkeitswirksam präsentiert wird. ${ }^{36} \mathrm{Im} \mathrm{Zu}$ sammenhang mit der nichtfinanziellen Berichterstattung führt die Praxis des Greenwashing bisher dazu, dass die von Unternehmen vorgelegten Berichte oft in weiten Teilen unglaubwürdig, nur schwer mit anderen Berichten vergleichbar und oftmals nicht umfassend und damit in der Praxis

36 Vgl. Boiral, Accounting, Auditing \& Accountability 2013 (26.7), 1036 (1061). Im Einzelnen handelt es sich dabei z.B. um die Verwendung von unklaren Begriffen bzw. suggestiven Bildern, die Verbreitung unvollständiger oder fehlerhafter Informationen, die Beschönigung oder Überbetonung bestimmter Umstände (siehe hierzu Horiuchi / Schuchard / Shea et al., Understanding and Preventing Greenwash, 2009; Gillespie, Consumer Policy Review 2008 (18.3), 79, 80 f.). 
nur von geringem Interesse sind. ${ }^{37}$ Kritisiert wird zudem, dass die Berichte regelmäßig eine Vielzahl unnötiger Informationen enthalten und gleichzeitig die wichtigen Informationen vermissen lassen. ${ }^{38}$

Bzgl. der Ausrichtung möglicher Durchsetzungs- oder Monitoringmechanismen kann zwischen Verfahren unterschieden werden, die erstens die Durchsetzung der Pflicht zur Berichterstattung, zweitens die Durchsetzung der Pflicht zu vorgabenkonformer und richtiger Berichterstattung und drittens ein Monitoring der Umsetzung menschenrechtlicher Vorgaben, wie sie sich z.B. aus den UNGP oder den OECD-Leitsätzen ergeben, zum Gegenstand haben.

Die Richtlinie gibt den Mitgliedstaaten zunächst die Pflicht zur Einführung verbindlicher Berichterstattung auf. Mit der in das HGB eingeführten Berichterstattungspflicht wurde diese Vorgabe in das deutsche Recht implementiert und mit den oben genannten Sanktionsmöglichkeiten wird die Durchsetzung der Berichtspflicht wirksam gewährleistet.

Darüber hinaus stellt sich die Frage, ob auch die Pflicht zur richtigen und umfassenden Berichterstattung wirksam durchgesetzt werden kann.

Als Mechanismen zur Durchsetzung der Pflicht zur richtigen und vorgabenkonformen Berichterstattung käme zunächst die externe Prüfung der Berichte in Betracht. Allerdings wurde oben bereits festgestellt, dass das Umsetzungsgesetz im Hinblick auf die externe Prüfung keine Pflicht zur Durchführung einer solchen vorsieht.

Das Durchsetzungsverfahren (Enforcementverfahren) im Bereich der finanziellen Berichterstattung umfasst indes nunmehr auch die nichtfinanzielle Erklärung bzw. den gesonderten nichtfinanziellen Bericht. In der Literatur wird zwar darauf verwiesen, dass die Prüfung durch die Deutsche Prüfstelle für Rechnungslegung e. V. (DPR) und die Bundesanstalt für Finanzdienstleistungsaufsicht (BaFin) systematisch auf die externe Prüfung der Berichte aufbaue und sich deshalb die Prüfung der DPR und der BaFin ebenfalls auf das Vorliegen einer Erklärung bzw. eines Berichts beschrän-

37 Leser_innen der Berichte haben deshalb darauf hingewiesen, dass angesichts der oftmals verfolgten Greenwashing-Strategien großes Misstrauen gegenüber den Intentionen von Unternehmen in Bezug auf deren Berichterstattung besteht (van Wensen / Broer / Klein et al., The State of Play in Sustainability Reporting in the European Union, 2011, S. 86).

38 European Coalition for Corporate Justice (ECCJ), Position Paper: EU Legislation on Non-financial Reporting by Companies, S. 1. 
ken würde. ${ }^{39}$ Allerdings wurde ein ausdrücklicher Ausschluss der nichtfinanziellen Berichterstattung von der Prüfung im Rahmen des Enforcementverfahrens nicht vorgenommen. Und auch dem Gesetzesentwurf der Bundesregierung ist kein Hinweis darauf zu entnehmen, dass die nichtfinanzielle Erklärung bzw. der gesonderte nichtfinanzielle Bericht nicht umfasst sein soll. Dies spricht dafür, dass auch die nichtfinanzielle Erklärung und der nichtfinanzielle Bericht mitumfasst sind.

Als Mechanismen zur Durchsetzung richtiger und vorgabenkonformer Berichterstattung kann zunächst auf das Sanktionsregime des HGB verwiesen werden. Denn dieses bezieht sich nicht nur auf die Frage der Berichterstattung, sondern sieht auch Sanktionen für Fälle fehlerhafter Berichterstattung vor. ${ }^{40}$ Darüber hinaus ist als weiterer Mechanismus die Prüfungspflicht des Aufsichtsrats zu nennen. ${ }^{41}$ Außerdem werden aktuell verschiedene Möglichkeiten des »private Enforcement«, d.h. der Durchsetzung richtiger und vorgabenkonformer Berichterstattung durch private Akteur_innen, diskutiert. ${ }^{42}$ In diesem Zusammenhang sind insbesondere die Frage der Haftung für unwahre Aussagen über Nachhaltigkeitskodizes im Rahmen des Kaufrechts, ${ }^{43}$ die Möglichkeit der Anfechtbarkeit von Hauptversammlungsbeschlüssen ${ }^{44}$ und die wettbewerbsrechtlichen Verbandsklagemöglichkeiten nach dem $\mathrm{UWG}^{45}$ von besonderem Interesse. Eine Umsetzung des in ErwG 10 formulierten Vorschlags, Verfahren einzurichten, die »allen natürlichen und juristischen Personen offenstehen (sollten), die gemäß nationalem Recht ein berechtigtes Interesse daran haben, dass sichergestellt wird, dass die Bestimmungen dieser Richtlinie eingehalten werden «, ist danach bisher nicht erfolgt. Hierzu müssten einschlägige NGOs mit einem umfassenden Verbandsklagerecht ausgestattet werden.

39 Kumm / Woodtli, DK 2016, 218 (231 f.); Seibt, DB 2016, 2707 (2714).

40 Siehe hierzu Brunk in diesem Band. Siehe auch Kumm / Woodtli, DK 2016, 218 (229 ff.).

41 Siehe hierzu Brunk in diesem Band.

42 Siehe hierzu ausführlich die Beiträge von Brunck, Horst und Segger in diesem Band.

43 Siehe hierzu Asmussen, NJW 2017, 118.

44 Siehe hierzu Horst in diesem Band.

45 Siehe hierzu Burckhardt, Die Schönfärberei der Discounter: Klage gegen Lidl's irreführende Werbung; Saage-Maaß / Müller-Hoff, Fairer Wettbewerb! Verbraucherklage in Deutschland für die Rechte der Arbeiterinnen in Südostasien, 2010. 
Neben der Durchsetzung der Berichtspflicht und der Pflicht zur richtigen und vorgabenkonformen Berichterstattung stellt sich drittens die Frage, wie sichergestellt werden kann, dass Unternehmen die Maßnahmen, über deren Umsetzung sie berichten - also z.B. die Durchführung von Due Diligence-Prozessen oder Impact Assessments - tatsächlich umsetzen. Hierbei geht es darum, wie die Umsetzung der z.B. in den UNGP oder den OECD-Leitsätzen genannten prozeduralen Vorgaben durchgesetzt werden kann und ob nichtfinanzielle Berichterstattung hierbei eine Rolle spielt, bzw. spielen kann. Eingangs wurde bereits darauf hingewiesen, dass nichtfinanzielle Berichterstattung darauf ausgerichtet ist, die Umsetzung der in den UNGP genannten prozeduralen Instrumente sicherzustellen. Allerdings ist diesbezüglich zu beachten, dass jedenfalls in Deutschland bisher keine gesetzlich geregelte Pflicht ${ }^{46}$ zur Beachtung dieser Vorgaben besteht. ${ }^{47}$ Insofern sind entsprechende Vorgaben den bisher auf privater oder internationaler Ebene verabschiedeten Regelungswerken zu entnehmen.

In den folgenden Abschnitten soll untersucht werden, welches Potential Berichtsverfahren aufweisen, die auf die Umsetzung menschenrechtlicher Vorgaben gerichtet sind (C. und D.) und wie entsprechende Verfahren im Verhältnis Wirtschaft \& Menschenrechte implementiert werden könnten (E.).

46 In Frankreich wurde hingegen erst kürzlich ein Gesetz verabschiedet, das vorsieht, dass den Unternehmen für eigene Aktivitäten und die ihrer Tochtergesellschaften und Zuliefer_innenbetriebe eine Vorsorgepflicht u.a. zur Vermeidung von Menschenrechtsverletzungen sowie eine damit zusammenhängende Veröffentlichungspflicht auferlegt werden soll (siehe hierzu Krebs, Wirtschaft und Menschenrechte: die „Loi Rana Plaza“ vor dem französischen Conseil constitutionnel, 29.03.2017, http://verfassungsblog.de/wirtschaft-und-menschenrechte-die-loi-rana-plaza-vordem-franzoesischen-conseil-constitutionnel/, (zuletzt abgerufen am 21.11.2017).

47 Ausführlich zu der Frage, ob das Unterlassen etwa von Due Diligence-Prozessen eine deliktische Haftung begründen kann siehe Heinen in diesem Band. Siehe auch siehe Wagner, RabelsZ 2016, 717; Weller / Kaler / Schulz, AcP 2016, 387; Osieka, Zivilrechtliche Haftung deutscher Unternehmen für menschenrechtsbeeinträchtigende Handlungen ihrer Zulieferer. 2013; Wesche / Saage-Maaß, HRLR 2016, 1; Grabosch, in: Nikol / Bernhard / Schmiederjahn (Hrsg.), Transnationale Unternehmen und Nichtregierungsorganisationen im Völkerrecht, 2013, S. 69; Massoud, Grabosch, in: Nikol / Bernhard / Schmiederjahn (Hrsg.), Transnationale Unternehmen und Nichtregierungsorganisationen im Völkerrecht, 2013, S. 69 (44 ff.); Hennings, Über das Verhältnis von multinationalen Unternehmen zu Menschenrechten, 2009, S. 125 ff.; Saage-Maaß, Arbeitsbedingungen in der globalen Zulieferkette, 2011, S. 7 ff. 


\section{Staatliche Menschenrechtsberichterstattung vor den UN- Menschenrechtsorganen}

Um die Stärken eines auf Berichterstattung setzenden Verfahrens beurteilen zu können, soll nun ein Blick auf die Praxis staatlicher Menschenrechtsberichterstattung vor den UN Menschenrechtsorganen geworfen werden. Die UN-Menschenrechtsabkommen sehen in der Regel vor, dass Staaten vor dem jeweiligen Überwachungsorgan über die Umsetzung der vertraglichen Vorgaben berichten müssen. ${ }^{48}$ Eine wesentliche Problemlage besteht dabei seit jeher darin, dass die Ausschüsse mit der steigenden Zahl der Vertragsstaaten sowie der im Laufe der Zeit steigenden Anzahl der periodischen Staatenberichte chronisch überlastet waren und ihrer Überprüfung oftmals nur mit Verspätung nachgekommen sind. Außerdem sieht sich die Praxis seit jeher weiterer erheblicher Kritik ausgesetzt. Die Verfahren gelten als »schwerfällig und teilweise redundant; für die unmittelbar Beteiligten sind sie mit einem hohen Arbeitsaufwand verbunden; für Uneingeweihte sind sie schlecht verständlich, für die Medien kaum von Interesse und in ihrer praktischen Bedeutung werden sie oft überschätzt. « ${ }^{49}$ Gusy fasst die Kritik wie folgt zusammen: Das Verfahren sei aufgrund seiner langen Dauer, fehlender Sanktions-, Kassations- und Rehabilitationsmöglichkeiten und der Überlastung der Überwachungsausschüsse durch die Papier- und Informationsflut ineffektiv. ${ }^{50}$

Ausgehend von dieser Kritik hat eine durch die Überwachungsausschüsse eingeleitete Reformierung des Berichtsprozesses stattgefunden. Besonders positiv zu werten ist dabei vor allem das von dem UN-Ausschuss gegen Folter seit langem praktizierte »Simplified Reporting Proce-

48 Zur Praxis der Berichtsverfahren siehe etwa Spenlé, Die Staatenberichtsverfahren der UNO-Menschenrechtsverträge, 2011; Levin, GWILR 2016, 315; Liese, Staaten am Pranger, 2006; Oberleitner, Menschenrechtsschutz durch Staatenberichte 1998; Müller, Menschenrechtsmonitoring, 2011 und die Beiträge in Gusy (Hrsg.), Grundrechtsmonitoring, 2011.

49 humanrights.ch, Reform der Staatenberichtsverfahren der UNO-Vertragsorgane: Dublin-Prozess, 07.08.2012, https://www.humanrights.ch/de/internationale-mensc henrechte/nachrichten/vertragsorgane/reform-staatenberichtsverfahren-dublin-proz ess (zuletzt abgerufen am 21.11.2017).

50 Gusy, in: Gusy (Hrgs.), Grundrechtsmonitoring, 2011, S. 18. 
dure« (SRP)..${ }^{51}$ Mit dem SRP wird insgesamt das Ziel verfolgt, die Effektivität des Verfahrens sowie die Qualität der Berichte und der Maßnahmen der Ausschüsse durch eine bessere Abstimmung zwischen Ausschuss und Staat zu fördern. Konkret geht es darum, die periodische Berichterstattung nicht auf alle von dem jeweiligen Vertragswerk umfassten Bereiche zu beziehen, sondern das Verfahren auf konkrete Problemlagen und strukturelle Mängel zu fokussieren. ${ }^{52}$ Dies soll vor allem durch die Stärkung des so genannten Follow-Up-Verfahrens geschehen, also dem Teil des Verfahrens, der an die Evaluierung des Erstbereichs eines Staates anknüpft, indem schon hier der Gegenstand der folgenden Berichterstattung im Wesentlichen auf Punkte beschränkt wird, die bereits zuvor im Verfahren von Relevanz bzw. Gegenstand von Kritik waren. Das Verfahren des SRP rückt damit den Kern des Berichtsverfahrens in den Mittelpunkt: den fortlaufenden konstruktiven Dialog.

Der Charakter dieser staatenbezogenen Berichtsverfahren unterscheidet sich maßgeblich von anderen Durchsetzungsmechanismen. Denn der Zweck der Verfahren besteht in erster Linie darin, die Vertragsstaaten bei der Umsetzung der in den Pakten enthaltenen Rechte zu unterstützen und sie dazu anzuhalten, selbst auf die Verwirklichung der Menschenrechte durch entsprechende politische und rechtliche Maßnahmen auf nationaler Ebene hinzuwirken..$^{53}$ Das Verfahren ist zudem auf Dauer angelegt und stellt einen ständig laufenden und »niemals abgeschlossenen Prozess«

51 Pillay, Strengthening the United Nations Human Rights Treaty Body System, 2012, S. 47 ff. Das SRP wurde von dem UN-Ausschuss gegen Folter 2007 im Rahmen der 38. Session angenommen (UN Committee Against Torture (CAT), Report of the Committee Against Torture: Annual Report to the Thirty-Seventh Session (6-24 November 2006) and the Thirty-Eighth Session (30 April-18 May 2007), UN Doc. A/62/44, Rn. 23 f.). Siehe auch den Bereicht des UN-Ausschuss gegen Folter über den Status und die Erfahrungen mit dem verkürzten Verfahren: $U N$ Committee Against Torture (CAT), Status of the Optional Reporting Procedure of the Committee Against Torture and Proposals for its Revision, 27.11.2011, UN Doc. CAT/C/47/2.

52 Vgl. UN Committee Against Torture (CAT), Report of the Committee Against Torture: Annual Report to the Thirty-Seventh Session (6-24 November 2006) and the Thirty-Eighth Session (30 April-18 May 2007), UN Doc. A/62/44, Rn. 23.

53 CESCR, Report on the Third Session, Annex III, General Comment No. 1, UN Doc. E/C.12/1989/5, Rn. 1; Gusy, in: Gusy (Hrsg.), Grundrechtsmonitoring, S. 11 (14). 
dar, ${ }^{54}$ in dessen Rahmen es - soweit die Vertragsstaaten das Verfahren mit einer gewissen Ernsthaftigkeit betreiben - zu einem »(langsamen) Prozess der Aneignung menschenrechtlicher Inhalte (kommt), der nicht mehr ohne weiteres rückgängig zu machen ist « ${ }^{55}$. Das Verfahren zielt damit auf einen Lernprozess ab, der eine Internalisierung der menschenrechtlichen Vorgaben in die Praxis und das »Bewusstsein« der Staaten, zum einen durch Selbstevaluation und zum anderen durch Evaluation der Überwachungsorgane unter Einbeziehung zivilgesellschaftlicher Akteur_innen, beabsichtigt. ${ }^{56}$ Folge des Verfahrens ist zudem die praxisorientierte Normpräzisierung und Rechtsauslegung im Rahmen der von den Ausschüssen veröffentlichten abschließenden Bemerkungen (Concluding Observations). ${ }^{57}$

Die Stärken der Berichtsverfahren lassen sich auch in Abgrenzung zum Individualbeschwerdeverfahren vor nationalen und internationalen Instanzen, wie z.B. dem Europäischen Gerichtshof für Menschenrechte, aufzeigen. Als zentraler Punkt ist hierbei die Unabhängigkeit des Berichtsverfahrens von Einzelfällen bzw. von konkreten Sachverhalten mit individuell Betroffenen zu nennen, die es ermöglicht, die gesamte »Performance« staatlicher Praxis in den Blick zu nehmen. Umfasst ist deshalb nicht nur eine Überprüfung, ob der Staat die menschenrechtlichen Verpflichtungen im Einzelfall einhält, sondern auch, ob es strukturelle Mängel in organisatorischer Hinsicht gibt, die für die Verwirklichung der Menschenrechte von Bedeutung sind. Der dialogische und fortlaufende Charakter des Verfahrens zielt in präventiver Hinsicht auch darauf $a b$, Missstände in der staatlichen Praxis im Hinblick auf Exekutive, Legislative und Judikative frühzeitig zu identifizieren oder sogar zu antizipieren und Maßnahmen zu ihrer Ausräumung oder Verhinderung anzustoßen. ${ }^{58}$ Ein weiterer Gesichtspunkt der Einzelfallunabhängigkeit, der die Qualität der Berichtsverfahren auszeichnet, ist die Möglichkeit, strukturelle und systemische Menschenrechtsproblemlagen in den Blick zu nehmen. Wesentliches Merkmal dieser ist es, dass nur schwerlich konkrete Individuen oder Gruppen als Opfer identifiziert werden können, die Kausalität zwischen Schädigung und

54 Spenlé / Skalski, Schweizer Zeitschrift für internationales und europäisches Recht 2012, 1 (15).

55 Davy, in: Gusy (Hrsg.), Grundrechtsmonitoring, 2011, S. 238 (255).

56 Vgl. Ebenda, S. 252 ff.; Oberleitner, Menschenrechtsschutz durch Staatenberichte, 1998, S. $62 \mathrm{ff}$.

57 Siehe hierzu Liese, Staaten am Pranger, 2006, S. $110 \mathrm{ff}$.

58 Siehe Müller, Menschenrechtsmonitoing, 2011, S. $386 \mathrm{ff}$. 
Schädigungshandlung nur schwer auszumachen ist und/oder eine komplexe Kumulation im Hinblick auf die Ursachen der Schädigung bzw. der schädigenden Akteur_innen vorliegt. ${ }^{59}$ Als letzter Gesichtspunkt der Einzelfallunabhängigkeit ist hervorzuheben, dass das Berichtsverfahren unabhängig von Beschwerden Einzelner oder von Gruppen betrieben wird. Dass sich eine internationale Instanz einer Sache annimmt, setzt voraus, dass überhaupt ein e Kläger in vorhanden ist, die Klage finanziert wird, der Instanzenzug durchlaufen wird und die generellen Zulässigkeitsvoraussetzungen erfüllt sind. ${ }^{60}$ Hier besteht die Möglichkeit, dass eine Problemlage im Berichtsverfahren von dem entsprechenden Ausschuss selbst aufgegriffen wird. In diesem Zusammenhang stehen die Verfahren vor den UN-Ausschüssen auch der Zivilgesellschaft in gewissem Maße offen. Durch die Einreichung von so genannten Schattenberichten ${ }^{61}$ kann die Aufmerksamkeit auf eine bestimmte Problemlage gerichtet werden oder zu einem bereits gegenständlichen Sachverhalt Stellung bezogen und damit Einfluss auf den Fortgang des Verfahrens ausgeübt werden. Die Bezugnahme auf einen konkreten Einzelfall und die aufwendige Planung und finanzielle Absicherung eines Individualbeschwerdeverfahrens ist nicht erforderlich. ${ }^{62}$

59 Eine ausführliche Analyse entsprechender Konstellationen am Beispiel der Subvention von Agrargütern im Lichte des Rechts auf Nahrung findet sich bei Kommer, Menschenrechte wider dem Hunger, 2016, S. $286 \mathrm{ff}$.

60 Müller, Menschenrechtsmonitoring, 2011, S. 388; Davy, in: Gusy (Hrsg.), Grundrechtsmonitoring, 2011, S. 238 (251).

61 Zur Rolle der NGOs bei den Berichtsverfahren siehe etwa Vandenhole, The Protection Before the UN Human Rights Treaty Bodies, 2004, S. 125 ff.; Brett, in: Alfredsson / Grimheden / Ramcharan et al. (Hrsg.), International Human Rights Monitoring Mechanisms, 2009, S. 673; Clapham, in: Alston / Crawford (Hrsg.), The Future of the UN Human Rights Treaty Monitoring, 2000, S. 175.

$62 \mathrm{Zu}$ den Herausforderungen der strategischen Prozessführung vor den UN-Fachausschüssen siehe Althoff, in: Kindermann / Schellenberg / Schons et al. (Hrsg.), Anwaltschaft für Menschenrechte und Vielfalt, 2014, S. 95; zu Herausforderungen der strategischen Prozessführung im Bereich Wirtschaft \& Menschenrechte siehe Saage-Maaß, Unternehmen zur Verantwortung ziehen: Erfahrungen aus transnationalen Menschenrechtsklagen, 2014. 


\section{Lernprozesse im Weltrecht und im Bereich Wirtschaft \& Menschenrechte}

Wie aber können die Erkenntnisse aus dem Bereich der staatlichen Menschenrechtsberichterstattung für die nichtfinanzielle Berichterstattung fruchtbar gemacht werden? Das Modell staatlicher Menschenrechtsberichterstattung auf die Praxis nichtfinanzieller Berichterstattung zu übertragen, würde die Besonderheiten des Verhältnisses von Menschenrechten \& Wirtschaft und nichtfinanzieller Berichterstattung außer Acht lassen. Es ist deshalb danach zu fragen, ob in der Praxis staatlicher Menschenrechtsberichterstattung generalisierbare Prinzipien $z u$ finden sind, die sich für den Bereich der nichtfinanziellen Berichterstattung respezifizieren lassen. ${ }^{63}$ Als entsprechendes Prinzip mit gesamtgesellschaftlicher Bedeutung lässt sich hier das für die Berichterstattungsverfahren zentrale Merkmal des Lernprozesses identifizieren, der im Rahmen des Verfahrens ein- und angeleitet werden soll.

Ein Blick auf die Beziehungen unterschiedlicher Regelungsbereiche auf Weltebene zeigt, dass, gerade im Verhältnis dieser zueinander, Lernprozesse eine wesentliche Rolle spielen. Als Beispiel hierfür soll auf das Verhältnis zwischen der Arbeit der OECD im Bereich Menschenrechte und transnationale Unternehmen verwiesen werden. Die OECD erarbeitet Standards, Richtlinien und Leitfäden für diesen Themenbereich und bietet sie den Unternehmen zur Nutzung an. Neben den OECD-Leitsätzen zählen hierzu z.B. die sektor-spezifischen Initiativen in den Bereichen »Agricultural Supply Chains«, »Extractive Sector Stakeholder Engagement«, »Financial Sector Due Diligence«, »Mineral Supply Chains« und »Textile and Garment Supply Chains«. Die Initiativen in all diesen Bereichen stellen sich so dar, dass die OECD - teilweise in enger Kooperation mit anderen Organisationen, wie z.B. der FAO - nach einem Ausarbeitungsprozess unter Einbeziehung relevanter Akteur_innen und nach einem Konsultationsprozess Standards und Richtlinien entwickelt oder bereits entwickelt hat, die von Unternehmen herangezogen werden können. ${ }^{64}$ Sie geben den

$63 \mathrm{Zu}$ Methode der Generalisierung und Respezifizierung siehe Teubner, ZaöRV 2003, 1 angewandt auf den Verfassungsbegriff im Kontext globaler Konstitutionalisierungsprozesse.

64 Für die genannten Bereiche handelt es sich dabei zunächst um die Leitlinien im Bereich Konfliktmineralien und „Textile and Garment Supply Chains": Organisation for Economic Co-operation and Development (OECD), OECD Due Dilli- 
Unternehmen Hilfestellungen zur vorgabenkonformen Organisation und Strukturierung des eigenen Unternehmens und zum vorgabenkonformen Handeln im Einzelfall.

Im Nebeneinander unternehmensinterner Regulierung und dem Regelungsbereich der OECD-Leitsätze findet nun zwar keine direkte Kommunikation bzw. kein direkter Transfer von Normen statt, sondern vielmehr wechselseitige Anregungen, die als wechselseitige Irritationen ${ }^{65}$ oder als Interpenetrationen ${ }^{66}$ beschrieben werden können. In diesem Sinne produziert das Regime der OECD-Leitsätze Normen in Form von Richtlinien, Empfehlungen und Prinzipien, »an denen die Unternehmen ablesen können, welche gesellschaftlichen Erwartungen an sie gestellt werden, ohne dass sie diese eins zu eins übernehmen müssen «. ${ }^{67}$ »Irritationen« erfolgen daher zunächst in der Formulierung und Bereitstellung entsprechender Bestimmungen und Instrumente. Diese werden als »kognitive Ressourcen« bereitstellt, um damit auf den Abstimmungsprozess der betreffenden Organisation zu externen sozialen Bereichen und der ökologischen Umwelt Einfluss zu nehmen. ${ }^{68}$ In der vorliegenden Konstellation werden Unternehmen kognitive Ressourcen zur Verfügung gestellt, die diese nutzen können (oder auch nicht), um ihre Umwelten »auszukundschaften« und um sie in die Lage zu versetzen, mit den dadurch gewonnenen Erkenntnissen ein menschenrechts- bzw. umweltkompatibles Verhalten herstellen zu

gence Guidance for Responsible Chains of Minerals from Conflict-Affected and High-Risk Areas, 2013 und Organisation for Economic Co-operation and Development (OECD), OECD Due Diligence Guidance for Responsible Supply Chains in the Garment and Footwear Sector, 2017 . In den Bereichen „Agricultural Supply Chains“ und „Extractive Sector Stakeholder Engagement" liegen bisher Entwürfe für entsprechende Leitlinien vor: Organisation for Economic Co-operation and Development (OECD) Draft Due Diligence Guidance for Meaningful Stakeholder Engagement in the Extractives Sector; Food and Agriculture Organization of the United Nations (FAO) und Organisation for Economic Co-operation and Development (OECD), Draft FAO-OECD Guidance for Responsible Agricultural Supply Chains, 2015. Im Bereich „Financial Sector Due Diligence“ wird die Ausarbeitung einer entsprechenden Leitlinie derzeit vorbereitet (siehe hierzu die Homepage der OECD).

65 Teubner, Festschrift für Klaus J. Hopt zum 70. Geburtstag, 2010, S. 1449.

66 Amstutz / Karava, Festschrift für Gunther Teubner zum 65. Geburtstag, 2009, S. 646.

67 Teubner, Festschrift für Klaus J. Hopt zum 70. Geburtstag, 2010, S. 1449 (1468).

68 Amstutz / Karava, Festschrift für Gunther Teubner zum 65. Geburtstag, 2009, S. 646, (657). 
können. ${ }^{69}$ Auf der anderen Seite werden die menschenrechtlichen Bestimmungen durch die Umsetzung und ihre praktische Anwendung durch ihre Adressat innen konkretisiert und fortentwickelt. In diesem Sinne ist das Verhältnis »wechselseitig«, weil das Rechtsregime der OECD-Leitsätze darauf ausgerichtet ist, seine kognitiven Ressourcen an die Verhältnisse und Entwicklungen in der Weltgesellschaft anzupassen. ${ }^{70}$ Und dadurch, dass im Wege dieser Interlegalität das OECD-Regime den Unternehmen kognitive Ressourcen zur Verfügung stellt, nimmt es Einfluss auf Lernprozesse in den Unternehmen, mit dem Ziel, die Adäquanz ihrer Aktivitäten $\mathrm{zu}$ anderen sozialen Bereichen zu steigern. ${ }^{71}$

Hierbei ist jedoch zu berücksichtigen, dass entsprechende Lernprozesse nur in eingeschränktem Maße von allein eingeleitet werden und nur die Unternehmen selbst darüber entscheiden, Veränderungsprozesse vornehmen. Deshalb sind externe Pressionen notwendig, die auf die eigenständige Anpassung z.B. von transnationalen Unternehmen auf andere soziale Belange ausgerichtet sind und rechtliche oder zivilgesellschaftliche Sanktionsmechanismen bereithalten, die geeignet sind, wirtschaftlichen Druck auszuüben. ${ }^{72}$ Im Bereich Wirtschaft \& Menschenrechte sind dies in erster Linie zivilgesellschaftliche Skandalisierungsprozesse, der Druck von Strategien im Bereich des nachhaltigen Konsums und Investments sowie politische Maßnahmen, die darauf ausgerichtet sind, Unternehmen zu nachhaltigerem Verhalten anzuhalten. Es zeigen sich aber auch Tendenzen, Unternehmen durch gesetzgeberische Maßnahmen darauf zu verpflichten, Menschenrechtsbelange zu berücksichtigen. Beispiele hierfür sind die hier gegenständliche Richtlinie 2014/95/EU sowie die in einigen Ländern bereits bestehende oder angestoßene Gesetzgebung im Hinblick auf Due Diligence-Prozesse. ${ }^{73}$

69 Ebenda, (661).

70 Ebenda, (664).

71 Ebenda, $(657,665$ f.).

72 Teubner, Verfassungsfragmente, 2012, S. 135, $146 \mathrm{ff}$.

73 Siehe hierzu die aktuellen Entwicklungen in Frankreich (hierzu Fn. 46) oder etwa Europäische Kommission, Vorschlag für eine Verordnung des Europäischen Parlaments und des Rates zur Schaffung eines Unionssystems zur Selbstzertifizierung der Erfüllung der Sorgfaltspflicht in der Lieferkette durch verantwortungsvolle Einführer von Zinn, Tantal, Wolfram, deren Erzen und Gold aus Konflikt- und Hochrisikogebieten, 05.03.2014, KOM(2014) 111 endgültig. Im April 2015 wurde zudem in der Schweiz die Konzernverantwortungsinitiative als Volksinitiative auf den Weg gebracht, die darauf abzielt, Due Diligence-Pflichten ins schweizerische 
Eine mit Monitoringverfahren ausgestattete nichtfinanzielle Berichterstattung kann hiernach ein Verfahren zur Umsetzung der Strategie der Einund Anleitung von Lernprozessen darstellen. Berichtsverfahren können als Kanal für wechselseitige Irritation dienen. Ein Überwachungsorgan kann Lernprozesse ein- und anleiten. In einem Verfahren zur Ein- und Anleitung von Lernprozessen geht es darum, die »Kompatibilisierung unterschiedlicher Teilrationalitäten sowie die Bevorratung gesamtgesellschaftlicher, struktureller Lernfähigkeit« herzustellen, ${ }^{74}$ indem rechtlich arrangierte Möglichkeiten für die Einforderung von Rechten und den Ausgleich widerstreitender Interessen bereitgestellt werden. ${ }^{75}$ In diesem Rahmen können wiederum eine Interpretation und Konkretisierung positiver Normierung sowie die Entwicklung einer teilbereichsspezifischen Dogmatik vorgenommen werden. ${ }^{76}$ Im Ergebnis kann jedoch erst dann, wenn Unternehmen auf die Kooperation mit entsprechenden (Monitoring-)Instanzen verpflichtet werden, sichergestellt werden, dass tatsächlich »ein Netzwerk rechtlicher Kontrolle entsteht, in dem Rechtsnormen wirksam werden «. ${ }^{77}$

\section{E. Perspektiven für ein Monitoring nichtfinanzieller Berichterstattung}

Wie, wo und von wem aber sollte bzw. könnte ein entsprechendes Modell verwirklicht werden? Die CSR-Strategie der EU setzt auf eine »intelligente Kombination« von Selbstregulierung und ergänzenden Legislativmaßnahmen. Ausgangspunkt dieses Ansatzes ist die Annahme, dass weder ein etatistischer Ansatz, der darauf setzt, Unternehmen mittels »verbindlicher « nationaler und internationaler Regulierung an Menschenrechte zu binden, noch ein Weg, der auf (Selbst-)Regulierung abseits des Staates setzt, sich als zielführend erweist. Denn ein etatistischer Ansatz verkennt erstens das Potenzial privater und internationaler »Soft Law«-Menschenrechtsregulierung im Hinblick auf die Produktion kognitiver Ressourcen, zweitens die rechtliche Wirkung, die solche Instrumente im Zusammenspiel mit ande-

Recht zu implementieren. Der Wortlaut der Volksinitiative ist abrufbar unter www.admin.ch/ch/d/pore/vi/vis462 t.html.

74 Calliess, Prozedurales Recht, 1999, S. 180.

75 Wiethölter, in: Joerges / Trubek (Hrsg.), Critical Legal Thought: An AmericanGerman Debate, 1989, S. 501 (510).

76 Ebenda.

77 Fischer-Lescano, JZ 2014, 965 (973). 
ren Regelungsbereichen entfalten können und drittens das Potenzial zivilgesellschaftlicher und privater Druck- und Durchsetzungsmöglichkeiten. Auf der anderen Seite erweisen sich auf Selbstregulierung setzende Strategien bzgl. der tatsächlichen Erfolge unternehmerischer Selbstregulierung regelmäßig als zu zuversichtlich. ${ }^{78}$ Sie lassen zudem Möglichkeiten unberücksichtigt, die staatliche Strukturen im Bereich der Durchsetzung bieten. Erfolgsversprechend erscheint daher ein Ansatz, der die jeweiligen Stärken staatlicher und privater Regulierung anerkennt und diese nicht als gegenläufigen oder unabhängig voneinander oder nebeneinanderlaufend begreift, sondern von einem durch Komplementarität und Interdependenz geprägten Verhältnis ausgeht

Eine Möglichkeit der Einführung von Monitoringmechanismen im Bereich der nichtfinanziellen Berichterstattung könnte darin bestehen, bereits bestehende Verfahren im Bereich Wirtschaft \& Menschenrechte auszuweiten. Als ein Anknüpfungspunkt könnten hierbei die Verfahren vor den im Rahmen der Umsetzung der OECD-Leitsätze eingerichteten Nationalen Kontaktstellen (NKS) dienen. ${ }^{79}$ Zudem wurde in der Praxis vorgeschlagen, beim Deutschen Institut für Menschenrechte (DIMR) eine Monitoringstelle für Streitfragen im Bereich Wirtschaft \& Menschenrechte einzu-

78 Die Wirtschaftskanzlei Eversheds hat 5000 Unternehmen kontaktiert und in ihrer Studie „Human Rights at Work 2016“ (Eversheds, On the Right Path: Human Rights at Work 2016 Report, 2016) konstatiert, dass nur 31\% der befragten Unternehmen Fortschritte bei der Umsetzung der UNGP machen würden. 43\% der Befragten Personen (general counsel, board directors and human resource directors) bescheinigten der Unternehmensführung ein mangelndes Engagement in Sachen Menschenrechten.

79 Eine ausführliche Analyse der Praxis der Nationalen Kontaktstellen findet sich bei Weidmann, Der Beitrag der OECD-Leitsätze für multinationale Unternehmen zum Schutz der Menschenrechte, 2014, S. 235 ff. Siehe hierzu auch folgenden Bericht der OECD zur Praxis der NKS zwischen 2000 und 2015: Organisation for Economic Co-operation and Development (OECD), Implementing the OECD Guidelines for Multinational Enterprises: The National Contact Points from 2000 to 2015, 2016. Eine Auswertung einiger Fälle vor den Nationalen Kontaktstellen der Schweiz, Großbritanniens und Deutschlands mit besonderem Augenmerk auf die Kriterien der Unparteilichkeit, Berechenbarkeit und Transparenz liefert das European Center for Constitutional and Human Rights (ECCHR) 2011. Siehe zudem Schmiederjahn, in: Nikol / Bernhard / Schmiederjahn (Hrsg.), Transnationale Unternehmen und Nichtregierungsorganisationen, 2013, S. 101 (105 ff.) sowie Krajewski / Bozorgzad / Heß, ZaöRV 2016, 309. 
richten. ${ }^{80}$ In der Folge sollen die Vor- und Nachteile dieser Möglichkeiten anhand einer kurzen Analyse der Verfahren vor den NKS eruiert werden.

Die OECD-Leitsätze sind Empfehlungen der OECD an multinationale Unternehmen und enthalten auf Freiwilligkeit beruhende Prinzipien in den Bereichen Arbeitsbedingungen, Menschenrechte, Umweltschutz, Transparenz, Korruption und Konsument_innenschutz. ${ }^{81}$ Sie sehen u.a. vor, dass die Mitgliedstaaten NKS einzurichten haben, die mit der Bearbeitung von Beschwerdeverfahren betraut sein sollen. ${ }^{82}$ Die Beschwerdeverfahren ${ }^{83}$ sind nicht als Klageverfahren, sondern eher als Diskussionsplattform ausgestaltet und darauf ausgerichtet, zwischen den Verfahrensbeteiligten zu vermitteln. In der "Verfahrensordnung" der OECD heißt es dementsprechend, dass $»[\mathrm{t}]$ he NCP will offer a forum for discussion and assist the business community, worker organisations, other non-governmental organisations, and other interested parties concerned to deal with the is-

80 Wissenschaftlicher Dienst des Bundestages, Anforderungen der VN-Leitprinzipien zu Wirtschaft und Menschenrechten an die Rechtsordnung in Deutschland, WD 2 $-3000-062 / 13,2013$, S. 16.

81 Eine Übersicht zur Entstehungsgeschichte, zur Ausgestaltung, zum aktuellen Stand nach der Neufassung der OECD-Leitsätze im Jahr 2011 und zu den Perspektiven liefern Huarte Melgar / Nowrot / Yuan, The 2011 Update of the OECD Guidelines for Multinational Enterprises, 2011; siehe ferner Tully, ICLQ 2001, 394; Johnston, in: Mullerat (Hrgs.), Coperate Social Responsibility: The Corporate Governance of the 21st Century, 2011, S. 275 ; Koeltz, Menschenrechtsverantworung multinationaler Unternehmen, 2010, S. $98 \mathrm{ff}$.

82 Organisation for Economic Co-operation and Development (OECD), OECD Guidelines for Multinational Enterprises - Procedural Guidance for NCPs, 2011, S. 68 .

83 Wichtige Hinweise zum Ablauf des Verfahrens liefern die Verfahrensordnung (ebenda), die Kommentierung hierzu (Organisation for Economic Co-operation and Development (OECD), OECD Guidelines for Multinational Enterprises Commentary on the Procedural Guidance for NCPs, 2011) sowie die Verfahrensordnungen der einzelnen NKS (siehe etwa die der deutschen NKS: Bundesministerium für Wirtschaft und Energie, Leitfaden zum Verfahren in besonderen Fällen („Beschwerde“) bei der deutschen Nationalen Kontaktstelle für die OECD-Leitsätze für multinationale Unternehmen,). Ausführlich hierzu mit Bezugnahme auf die Praxis der Nationalen Kontaktstellen: Weidmann, Der Beitrag der OECD-Leitsätze für multinationale Unternehmen zum Schutz der Menschenrechte, 2014, S. 237-242. 
sues raised in an efficient and timely manner and in accordance with applicable law $\ll^{84}$.

Den Mitgliedstaaten wird bei der Organisation der NKS und im Hinblick auf die Ausgestaltung der Beschwerdeverfahren ein Rahmen vorgegeben, der ihnen weitgehende Gestaltungsspielräume überlässt. Die Verfahrensordnung überlässt es den Staaten insbesondere, die NKS entweder an einer Behörde anzusiedeln oder diese als unabhängige Instanz auszugestalten. ${ }^{85}$ Die deutsche nationale Kontaktstelle ist daher z.B. beim Bundesministerium für Wirtschaft und Energie angesiedelt und besteht aus einer öffentlichen Dienststelle unter Leitung von Beamt_innen. ${ }^{86}$

Das Verfahren an sich verläuft generell in drei Schritten bzw. Phasen. ${ }^{87}$ Eingeleitet wird es durch die Erhebung einer »Beschwerde« bei der jeweiligen NKS. Die Anforderungen der OECD-Leitsätze an die persönlichen Voraussetzungen möglicher Beschwerdeführer_innen (»Beschwerdefähigkeit«) sind sehr weit gefasst, ${ }^{88}$ was dazu führt, dass nicht nur betroffene Individuen, sondern auch Gewerkschaften, NGOs oder betroffene Gruppen grds. beschwerdefähig sind. Nachdem eine »Beschwerde« eingereicht worden ist, beginnt die erste Phase des eigentlichen Verfahrens, in der es darum geht, im Rahmen eines »Initial Assessments« zu prüfen, ob die auf-

84 Organisation for Economic Co-operation and Development (OECD), OECD Guidelines for Multinational Enterprises - Procedural Guidance for NCPs, 2011, S. 72.

85 Ebenda, S. 71.

86 Die Ansiedlung der deutschen Kontaktstelle im Bundesministerium für Wirtschaft stellt einen wesentlichen Kritikpunkt an der Praxis in Deutschland dar. Es wird argumentiert, dass durch die organisatorische Ausgestaltung die Unabhängigkeit der Kontaktstelle nicht gewährleistet ist. Siehe hierzu European Center for Constitutional and Human Rights (ECCHR), Zwangs- und Kinderarbeit in Usbekistan: Wie effektiv ist der OECD-Beschwerdemechanismus?, 2013; Germanwatch, UN-Beauftragter kritisiert Anwendung der OECD-Leitsätze: Optimierung und Neustrukturierung der deutschen Nationalen Kontaktstelle für die OECD-Leitsätze erforderlich, 2008. A.A. Kasolowsky / Voland, NZG 2014, 1288 (1292), die insbesondere darauf verweisen, dass die deutsche NKS bereits mehrere Unternehmen gerügt hat, seine Entscheidungen mit anderen Ministerien abstimmt und ablehnende Entscheidungen ausreichend begründet.

87 Zum Ablauf der Verfahren vor der deutschen NKS siehe etwa Kasolowsky / Voland, NZG 2014, 1288.

88 Siehe hierzu Organisation for Economic Co-operation and Development (OECD), OECD Guidelines for Multinational Enterprises - Procedural Guidance for NCPs, 2011, S. 72 und Weidmann, Der Beitrag der OECD-Leitsätze für multinationale Unternehmen zum Schutz der Menschenrechte, 2014, S. 238. 
geworfene Frage eine eingehende Prüfung rechtfertigt. ${ }^{89}$ Eine Beschwerde ist hier insbesondere auch dann »zulässig «, wenn es nicht um einen konkreten Einzelfall geht, sondern um eine generelle Unternehmenspraxis, die potenziell schädliche Auswirkungen haben kann. Erforderlich ist nur, dass ein Zusammenhang zwischen der gegenständlichen Problemlage und der generellen Geschäftstätigkeit eines Unternehmens besteht. ${ }^{90} \mathrm{Im}$ Anschluss an das Initial Assessment folgt die zweite Phase des Verfahrens, in der es darum geht, den beteiligten Parteien durch die Bereitstellung von »good offices« Hilfe bei der Lösung der gestellten Frage anzubieten und auf eine einvernehmliche Lösung des Problems hinzuwirken. ${ }^{91}$ Die Gestaltungsmöglichkeiten der NKS treten vor allem in der dritten Phase hervor, in der es um die Beendigung des Verfahrens geht. Je nach Verfahrenskonstellation sollen die Nationalen Kontaktstellen (1.) in den Fällen, in denen sie der Meinung sind, dass ein Verfahren nicht zu eröffnen ist, eine Erklärung veröffentlichen, in der zumindest die aufgeworfenen Fragen sowie die Gründe für die Entscheidung der nationalen Kontaktstelle dargelegt sind. ${ }^{92}$ Soweit die Parteien eine Einigung getroffen haben, sollen die nationalen Kontaktstellen (2.) einen Bericht hierzu veröffentlichen, der u.a. auch mit ausdrücklicher Zustimmung der Parteien den Inhalt der Einigung darlegen soll. ${ }^{93}$ Wenn die Parteien keine Einigung erzielen konnten oder sich eine Partei weigerte, an dem Verfahren teilzunehmen, sollen die nationalen Kontaktstellen (3.) eine Erklärung über die in dem Verfahren aufgeworfenen Fragen abfassen, ggf. Empfehlungen zur Umsetzung der Leitsätze formulieren und ggf. in der Erklärung die Gründe aufnehmen, die eine Eini-

89 Organisation for Economic Co-operation and Development (OECD), OECD Guidelines for Multinational Enterprises - Procedural Guidance for NCPs, 2011, S. 72 .

90 Vgl. hierzu Weidmann, Der Beitrag der OECD-Leitsätze für multinationale Unternehmen zum Schutz der Menschenrechte, 2014, S. 242.

91 Organisation for Economic Co-operation and Development (OECD), OECD Guidelines for Multinational Enterprises - Procedural Guidance for NCPs, 2011, S. $72 \mathrm{f}$.

92 Ebenda, S. 73; Organisation for Economic Co-operation and Development $(O E C D)$, OECD Guidelines for Multinational Enterprises - Commentary on the Procedural Guidance for NCPs, 2011, S. 84, Rn. 32.

93 Organisation for Economic Co-operation and Development (OECD), OECD Guidelines for Multinational Enterprises - Procedural Guidance for NCPs, 2011, S. 73 . 
gung verhindert haben. ${ }^{94}$ Das Verfahren sieht keine Sanktionsmöglichkeiten oder sonstige Durchsetzungsmöglichkeiten vor, weshalb die NKS darauf angewiesen sind, dass die Parteien an dem Verfahren teilnehmen und auf die Anregungen und Vorschläge der NKS eingehen bzw. diese umsetzen.

Das Verfahren vor den NKS ist danach zwar nicht als Berichtsverfahren ausgestaltet, weist aber Charakteristika und Fortentwicklungsmöglichkeiten im Hinblick auf die oben identifizierten Stärken der auf Berichterstattung setzenden Verfahren auf.

Zunächst bietet das Verfahren vor den NKS die Möglichkeit, strukturelle Problemlagen unabhängig von konkreten Schädigungshandlungen zu behandeln. In einem Verfahren gegen das Bergbauunternehmen Vedanta Sources plc., in dem von einer NGO gerügt wurde, dass das Unternehmen im Rahmen eines Mienenprojektes es versäumt habe, die von dem Projekt betroffene einheimische, indigene Bevölkerung zu konsultieren und zu berücksichtigen, hat die zuständige Nationale Kontaktstelle des Vereinigten Königreichs in dem Unterlassen der Konsultation und der fehlenden Durchführung eines Impact Assessments eine Verletzung der OECD-Leitsätze festgestellt. ${ }^{95}$ Die Kontaktstelle sieht darin nicht nur eine Verletzung der OECD-Leitsätze, ${ }^{96}$ sondern verweist auch auf internationale Menschenrechtsinstrumente (u.a. auf den UN-Zivilpakt und die UN-Erklärung

94 Ebenda; Organisation for Economic Co-operation and Development (OECD), OECD Guidelines for Multinational Enterprises - Commentary on the Procedural Guidance for NCPs, 2011, S. 85, Rn. 36.

95 Zur fehlenden Konsultation und der versäumten Durchführung eines Impact Assessments siehe UK National Contact Point for the OECD Guidelines for Multinational Enterprises, Final Statement v. 25.9.2009 (Survival International./. Vedanta Resources plc.), Rn. 60, abrufbar unter www.oecdwatch.org/cases/Case_165.

96 Konkret benannt werden Verletzungen der Chapter II 2. („Respect the human rights of those affected by their activities consistent with the host government's international obligations and commitments.“), Chapter II 7. („Develop and apply effective self-regulatory practices and management systems that foster a relationship of confidence and mutual trust between enterprises and the societies in which they operate.") und Chapter V 2. b (,engage in adequate and timely communication and consultation with the communities directly affected by the environmental, health and safety policies of the enterprise and by their implementation.") der 2000erFassung der OECD-Guidelines (UK National Contact Point for the OECD Guidelines for Multinational Enterprises, Final Statement v. 25.9.2009 (Survival International./. Vedanta Resources plc.), Rn. 64-67, abrufbar unter www.oecdwatch.org/c ases/Case_165). 
über die Rechte indigener Völker $)^{97}$ aus denen sich eine staatliche Pflicht zur Konsultation indigener Bevölkerungsgruppen ergebe, welche auch von Unternehmen zu respektieren sei. ${ }^{98}$ Neben der Formulierung konkreter Rechte der indigenen Bevölkerung sowie daraus resultierende $»$ Respektierungspflichten«, hat die NKS Empfehlungen abgegeben, die sich nicht auf den konkreten Sachverhalt beschränken, sondern sich auch auf die generellen Strukturen des Unternehmens beziehen. Die NKS benennt in diesem Sinne zu ergreifende Maßnahmen, wie z.B. die wirksame, d.h. vor allem praxisorientierte, Implementierung von Due Diligence-Prozessen in die Unternehmensstrukturen, ohne hierbei jedoch auf die konkreten Strukturen des Unternehmens einzugehen. ${ }^{99}$ Der Fall verdeutlicht, dass im Rahmen der Verfahren generell die Strukturen und Abläufe in Unternehmen Gegenstand der Prüfung sind, das Verfahren unabhängig von individuell Betroffenen geführt werden kann und die Anforderungen der OECD-Leitsätze konkretisiert werden.

Die Verfahren vor den NKS sehen sich jedoch auch - nicht unberechtigter - Kritik ausgesetzt. Kritisiert werden im Einzelnen die schlechte »Bilanz der Verfahren « ${ }^{100}$, Schwierigkeiten im Hinblick auf den Zugang zum Verfahren, die Unabhängigkeit der NKS, die Transparenz und Vorhersehbarkeit der Verfahren und Mängel bzgl. der Benennung und fortlaufenden Bearbeitung von Verstößen gegen die OECD-Leitsätze. ${ }^{101}$

Von (staatlichen) Berichterstattungsverfahren inspirierte Fortentwicklungsperspektiven lassen sich auch unter Heranziehung der Kritik an den Verfahren im Hinblick auf folgenden Fälle formulieren: Bei dem ersten Fall handelt es sich um ein Verfahren, in dem es um die Mitverantwortung von Textilunternehmen für den Brand in der Fabrik von Tazreen Fashion Ltd. in Bangladesch am 24. November 2012 ging. Neben Vorwürfen, die mit dem Brandereignis unmittelbar zusammenhingen, wurde den Unternehmen auch vorgeworfen, ihrer Sorgfaltspflicht zur Ermittlung und Ver-

97 Ebenda, Rn. 59.

98 Ebenda, Rn. 60.

99 Ebenda, Rn. 75-80.

100 Ein aktueller Bericht von OECD Watch bezieht im Hinblick auf die Effektivität der Verfahren vor den NKS wie folgt Stellung; „Of the 250 cases we examined, only three - a mere one per cent - led to an actual improvement in conditions for victims of corporate abuse. Only a further thirteen per cent had any sort of beneficial result, such as an improved corporate policy" (Daniel / Wilde-Ramsing / Genovese et al., Remedy Remains Rare, 2015, S. 19).

101 Ebenda, S. 21 ff.. 
meidung von negativen Auswirkungen ihrer Geschäftstätigkeit auf die Sicherheit und Gesundheit der Mitarbeiter_innen von Tazreen Fashion entgegen II. Nr. 10 und 13 sowie IV. Nr. 5 der OECD-Guidelines nicht nachgekommen zu sein. ${ }^{102} \mathrm{Im}$ weiteren Verlauf des Verfahrens, insbesondere des Vermittlungsverfahrens, lag der Fokus auf der Frage der Einhaltung von Sorgfaltspflichten. Der Beschwerdeführer hat jedoch - im Gegensatz zur deutschen Nationalen Kontaktstelle - bzgl. des Unternehmens KiK Textilien und Non-Food GmbH keine Grundlage für eine gütliche Einigung gesehen, da KiK dem Thema der Sorgfaltspflichten und der Einhaltung des eigenen Codes of Conduct nicht die nötige Aufmerksamkeit entgegengebracht habe, die Nachhaltigkeitsberichterstattung an entscheidenden Stellen unklar bleibe und die vorgeschlagenen Maßnahmen insgesamt nicht ausreichend seien. ${ }^{103}$ Die nationale Kontaktstelle beendete daraufhin das Verfahren und führte aus, dass »es begrüßenswert gewesen wäre, wenn $\mathrm{KiK}$ die geplanten Maßnahmen weiter konkretisiert hätte «104. Das Verfahren habe aber nicht weitergeführt werden können, da der Beschwerdeführer signalisiert habe, dass dieses Ziel im Rahmen eines Dialoges zwischen den Parteien nicht mehr zu erreichen sei. ${ }^{105}$

Die streng kontradiktorische Ausgestaltung des Verfahrens räumt der NKS danach nur wenige Handlungsspielräume für den Fall ein, dass eine Partei den Prozess nicht fortführen will. Eine Leitung des Verfahrens durch die Kontaktstelle (so wie in den Berichtsverfahren vor den UNMenschenrechtsorganen) hätte den Vorteil, dass diese unabhängig von dem Betreiben des Verfahrens durch die Beschwerdeführer_innen eine Evaluation von Strukturen und Maßnahmen im Bereich der Sorgfaltspflichten vornehmen könnte. Da die sorgfaltspflichtbezogenen Vorgaben oftmals einen längerfristigen Umsetzungs- und Implementierungsprozess erfordern, bestünde bei einer Leitung des Verfahrens durch die NKS die Möglichkeit, diesen (Lern-)Prozess (unter Einbeziehung zivilgesellschaftlicher Akteur_innen) zu begleiten und entsprechende Empfehlungen aus-

102 Deutsche Nationale Kontaktstelle für die OECD-Leitsätze für multinationale Unternehmen, Abschließende Erklärung v. 4.11.2014 (Uwe Kerkeritz (MdB)./. Kik Textilien und Non-Food GmbH, C\&A Mode GmbH \& Co und Karl Rieker GmbH \& Co. KG), S. 2, abrufbar unter www.bmwi.de/BMWi/Redaktion/PDF/A/ abschlusserklaerung-kekeritz,property $=$ pdf,bereich $=$ bmwi2012, sprache $=\mathrm{de}, \mathrm{rwb}=\mathrm{t}$ rue.pdf.

103 Ebenda, S. 10.

104 Ebenda, S. 11.

105 Ebenda. 
zusprechen. Dies hätte im zuvor benannten Fall die Möglichkeit geboten, die Praxis des Unternehmens kritisch zu begleiten. Eine entsprechende Vorgehensweise ist auch ausdrücklich im Leitfaden der deutschen Kontaktstelle vorgesehen, allerdings nur für den Fall, dass beide Parteien ein solches Monitoring wünschen. 106

Die Schwäche der Durchführung des Verfahrens ohne Beteiligungsbereitschaft der Beschwerdeführer_innen zeigt auch ein Verfahren, das vor der kanadischen NKS anhängig war. In der Sache FREDEMI coalition./. Goldcorp haben die Beschwerdeführer_innen, eine Gruppe guatemalischer Gemeinden, eine weitere Teilnahme an dem Dialog mit dem betroffenen Unternehmen ausgeschlossen, weil sie u.a. eine Verschärfung des bestehenden Konfliktes befürchteten. Sie haben die NKS ausdrücklich aufgefordert, das Verfahren ohne die aktive Beteiligung der Beschwerdeführer_innen fortzusetzen und insbesondere den Sachverhalt aufzuklären und eine abschließende Erklärung mit Empfehlungen zur Verwirklichung der Vorgaben der OECD-Guidelines abzugeben. ${ }^{107}$ Die kanadische NKS ist dieser Aufforderung indes nicht nachgekommen und hat das Verfahren abgeschlossen, ohne auf die Aufforderung der Beschwerdeführer_innen einzugehen. ${ }^{108}$

Hiernach kann festgehalten werden, dass es erstens der Wirksamkeit der Verfahren im Wege steht, dass die NKS nicht über die Kompetenz verfügen, das Verfahren unabhängig vom Willen der Parteien fortzuführen und zu leiten. Zweitens sind häufig und maßgeblich strukturelle Problemlagen Gegenstand der Verfahren, die nur angemessen im Rahmen eines fortlaufenden Verfahrens (Follow-Up) behandelt werden können. Diesbezüglich scheint es naheliegend, die Stärken der Berichtsverfahren vor den UN-Menschenrechtsausschüssen, die Ein- und Anleitung von Lernprozessen im Rahmen eines fortlaufenden Verfahrens, in den Verfahren vor den NKS zu implementieren. Ausgangspunkt und Referenzpunkt der Prüfung kann der nichtfinanzielle Bericht eines Unternehmens sein.

106 Bundesministerium für Wirtschaft und Energie, Leitfaden zum Verfahren in besonderen Fällen („Beschwerde“) bei der deutschen Nationalen Kontaktstelle für die OECD-Leitsätze für multinationale Unternehmen, S. 5.

107 Siehe hierzu Ochoa Sanchez, Nordic Journal of International Law 2015, 89 (101 ff). sowie die Dokumentation des Verfahrens durch OECD Watch, abrufbar unter www.oecdwatch.org/cases/Case_172.

108 Ebenda. 
Aufgrund der hohen Anzahl berichtspflichtiger Unternehmen erscheint es allerdings unmöglich, (wie bei den UN-Menschenrechtsorganen) für jedes Unternehmen ein Verfahren durchzuführen. In Bezug auf dieses Problem lohnt sich ein Blick auf die deutsche Monitoringpraxis im Bereich der finanziellen Berichterstattung. ${ }^{109}$ Gemäß $\S 342$ b Abs. 2 Satz 3 HGB wird dort in drei verschiedenen Konstellationen ein Verfahren eingeleitet: Wenn konkrete Anhaltspunkte für einen Verstoß gegen Rechnungslegungsvorschriften vorliegen (Nr. 1), wenn die Bundesanstalt für Finanzdienstleistungsaufsicht (BaFin) die Prüfung verlangt (Nr. 2) oder auch ohne besonderen Anlass im Rahmen einer stichprobenhaften Prüfung (Nr.3). Eine entsprechende Ausgestaltung würde Monitoringorgane entlasten und gleichzeitig die Möglichkeit bereithalten, aus konkretem Anlass ein Verfahren einzuleiten.

Der häufig geäußerten Kritik bzgl. der Ansiedlung der deutschen NKS beim Bundeswirtschaftsministerium ${ }^{110}$ könnte dadurch begegnet werden, die deutsche NKS beim DIMR anzusiedeln. Denn hierbei handelt es sich um eine gänzlich unabhängige Institution. ${ }^{111}$

Ein Vorteil, den ein Verfahren vor den NKS gegenüber staatlicher Menschenrechtsberichterstattung hätte, wäre der, dass einer NKS grundsätzlich nationalstaatliche Durchsetzungs- und Sanktionsmechanismen übertragen werden könnten. Im Gesetzgebungsverfahren zur Umsetzung der Richtlinie 2014/95/EU hat Felber (Mitbegründer des Wirtschaftsmodells »Gemeinwohl-Ökonomie «) in diesem Zusammenhang folgende Druckmittel bzw. Bevorzugungsmöglichkeiten aufgeführt, die im Rahmen der Verfahren angeordnet oder eingeleitet werden könnten:

109 Siehe hierzu ausführlich Bockmann, Internationale Koordinierung nationaler Enforcement-Aktivitäten, 2012; Lohwasser, Effizienz der Kapitalmärkte durch Enforcement von IFRS, 2006.

110 Siehe Fn. 86.

111 Gemäß $\S 1$ des Gesetzes über die Rechtsstellung und Aufgaben des Deutschen Instituts für Menschenrechte vom 16.7.2015 (BGB1. I S. 1194) ist das DIMR die unabhängige nationale Institution der Bundesrepublik Deutschland zur Information der Öffentlichkeit über die Lage der Menschenrechte im In- und Ausland sowie zur Förderung und zum Schutz der Menschenrechte. Laut $\S 2$ Abs. 1 S. 2 handelt es unabhängig von Vorgaben und Weisungen der Bundesregierung oder anderen öffentlichen und privaten Stellen in eigener Initiative oder auf Ersuchen der Bundesregierung oder des Deutschen Bundestages unter eigenverantwortlichem Einsatz seiner Ressourcen. 
- Sanktionen (Geldstrafen)

- Vorteile/Nachteile bei Subventions- oder sonstigen Unterstützungsleistungen

- Vorrang/Nachrang in der öffentlichen Beschaffung

- Differenzieren bei Steuersätzen

- Differenzieren bei Marktzugangsschwellen (z. B. Zölle)

- Differenzieren bei Kreditkonditionen (ethische Bonität)

Die kanadische NKS hat in einem Verfahren (Canada Tibet Committee./. China Gold International Resources Corp. Ltd.) sogar ausdrücklich entsprechende Sanktionen verhängt, weil das Unternehmen die Teilnahme an dem Verfahren verweigert hat. ${ }^{112}$ Solange das Unternehmen die Teilnahme an dem Verfahren verweigert, behalten sich die kanadischen Behörden vor, Unterstützungsleistungen des kanadischen Trade Commissioner Services und sonstige kanadische Hilfsmaßnahmen dem Unternehmen vorzuenthalten.

Auf den ersten Blick erscheint die Ansiedlung der NKS beim DIMR diesbezüglich nachteilhaft, da dieses selbst keine hoheitlichen Maßnahmen einleiten bzw. anordnen dürfte. Auch hier könnte jedoch wieder das deutsche Monitoringverfahren im Bereich der finanziellen Berichterstattung als Vorbild dienen. Dieses ist zweistufig organisiert und besteht aus dem privatrechtlich organisierten Verein Deutsche Prüfstelle für Rechnungslegung DPR e.V. (DPR) sowie der (der Rechts- und Fachaufsicht des Bundesfinanzministeriums unterstehenden) Bundesanstalt für Finanzdienstleistungsaufsicht (BaFin). Während die DPR selbst nur die Prüfung der Berichterstattung vornimmt, kommt nur der BaFin die Kompetenz zu, über Sanktionen und sonstige Anordnungen zu entscheiden. Ein ähnliches Zusammenspiel wäre auch für das Verhältnis zwischen dem DIMR und sonstigen staatlichen Instanzen denkbar. Insofern würde mit der Ansiedlung der NKS beim DIMR bzw. der Einrichtung einer Monitoringstelle beim DIMR gegenüber der jetzigen Lösung im Hinblick auf Durchset-

112 In ihrer abschließenden Stellungnahme vom 1.4.2015 heißt es: „As the Company did not respond to the NCP's offer of its good offices, the Company's non-participation in the NCP process will be taken into consideration in any applications by the Company for enhanced advocacy support from the Trade Commissioner Service and/or Export Development Canada (EDC) financial services, should they be made." (National Contact Point Canada, Final Statement v. 1.4.2015 (Canada Tibet Committee./. China Gold International Resources Corp. Ltd.), S. 2, 8, abrufbar unter www.oecdwatch.org/cases/Case_324). 
zungs- und Sanktionsmechanismen grds. keine Nachteile entstehen. Besonders im Hinblick auf die Unabhängigkeit des DIMR erscheint dieses nach alledem als besonders geeignet, das zuvor skizzierte Monitoring $\mathrm{zu}$ betreiben. 